\title{
1 Prediction and validation of host cleavage targets of SARS-CoV-2 3C- 2 like protease
}

Nora Yucel ${ }^{1}$, Silvia Marchiano ${ }^{2,3,4}$, Evan Tchelepi ${ }^{5}$, Germana Paterlini ${ }^{6}$, Quentin McAfee ${ }^{1}$, Nehaar Nimmagadda ${ }^{1}$, Andy Ren ${ }^{1}$, Sam Shi ${ }^{1}$, Charles Murry ${ }^{2,3,4,7,8,9}$, Zoltan Arany ${ }^{1}$

${ }^{1}$ Cardiovascular Institute, Perelman School of Medicine, University of Pennsylvania, Philadelphia PA 19104

${ }^{2}$ Institute for Stem Cell and Regenerative Medicine, University of Washington, 850 Republican Street, Brotman Building Room 453, Seattle, WA 98109, USA.

${ }^{3}$ Center for Cardiovascular Biology, University of Washington, Seattle, WA 98109, USA

${ }^{4}$ Department of Laboratory Medicine \& Pathology, University of Washington, Seattle, WA 98195, USA

${ }^{5}$ NetQuest Corporation, 523 Fellowship Rd Suite 205, Mt Laurel Township, NJ 08054

${ }^{6}$ Certusoft, 8500 Normandale Blvd, \#980, Bloomington, MN, 55437

${ }^{7}$ Division of Cardiology, Department of Medicine, University of Washington, Seattle, WA 98195, USA

${ }^{8}$ Sana Biotechnology, Seattle, WA 98102, USA

${ }^{9}$ Department of Bioengineering, University of Washington, Seattle, WA 98195, USA

Corresponding:

Zoltan Arany MD PhD

Professor in Medicine

Perelman School of Medicine

University of Pennsylvania

Smilow Center for Translational Research

11th floor

3400 Civic Blvd

Philadelphia 19104

Administrative assistant:

Emily Romick

\section{Abstract}

How SARS-CoV-2 causes the observed range of clinical manifestations and disease severity

remains poorly understood. SARS-CoV-2 encodes for two proteases (3CLPro and PLPro), vital for

viral production, but also promiscuous with respect to host protein targets, likely contributing

to the range of disease. Pharmacological inhibition of the 3C-like3 protease has revealed

remarkable reduction in hospitalization and death in phase $2 / 3$ clinical studies. However, the 
41 mechanisms responsible for the pathology mediated by those proteases are still unclear. In this

42 study, we develop a bioinformatic algorithm, leveraging experimental data from SARS-CoV, to

43 predict host cleavage targets of the SARS-CoV-2 3C-like protease, or 3CLPro. We capture targets

44 of the $3 \mathrm{CL}$ protease described previously for SARS-CoV, and we identify hundreds of new

45 putative targets. We experimentally validate a number of these predicted targets, including the

46 giant sarcomeric protein Obscurin, and show that expression of $3 \mathrm{CL}$ protease alone

47 recapitulates the sarcomeric disorganization seen by SARS-CoV-2 infection of hiPSC-derived

48 cardiomyocytes. Our data provide a resource to identify putative host cleavage targets of $3 \mathrm{CL}$

49 protease that contribute to mechanisms and heterogeneity of disease in COVID-19 and future

50 coronavirus outbreaks.

\section{Introduction}

53 COVID-19 continues to be a leading cause of death and morbidity across the world since the

54 initial outbreak in Wuhan, China December 2019¹. How SARS-CoV-2, the causative agents of

55 COVID19, leads to its wide range of disease manifestations remains incompletely understood.

56 In addition to lung damage, SARS-CoV-2 infection can also cause kidney damage, clotting

57 disorders, loss of taste and smell, cognitive dysfunction, muscle atrophy, and cardiac

58 dysfunction ${ }^{2-5}$. In addition, long-lasting COVID-19 symptoms have been reported in patients up

59 to a year after initial illness, including fatigue, shortness of breath, brain fog, and elevated heart

60 rate ${ }^{6,7}$. It remains unclear how SARS-CoV-2 affects differently the multiple organs and cell types

61 involved. Nor it is known what host characteristics determine who will develop severe COVID- 
6219, which organs will be affected, or whether long COVID will ensue. A deeper mechanistic

63 understanding of virus-host interactions is thus needed.

65 Various studies have identified host interaction partners of many SARS-CoV-2 proteins,

66 including the spike, envelope, nucleocapsid, and membrane proteins ${ }^{8-10}$. These interactions

67 have various consequences, including suppression of innate immune response, suppression of

68 apoptosis, and reprogramming of host transcription and translation. In addition to protein-

69 protein interactions, important virus-host interactions can be caused by enzymatic cleavage of

70 host proteins by viral proteases. For example, myocardial dysfunction following infection by

71 coxsackie CVB3 virus can in part be ascribed to cleavage of dystrophin protein by the viral

72 protease $2 \mathrm{~A}^{11,12}$; enteroviral $3 \mathrm{C}$ proteases can cleave host NLRP1 to trigger inflammasome

73 activation ${ }^{13}$; HIV-1 protease mediates apoptosis by cleaving host procaspase 9 and $\mathrm{Bcl} 2^{14,15}$;

74 and the Zika virus nsP2 cysteine protease can cleave host proteins SFRP1 NT5M, and FOXG1 ${ }^{16}$.

75

76 SARS-CoV-2 encodes for two proteases, a papain-like protease (PLPro) and the 3C-like protease

77 (3CLPro, also knowns as main Protease, MPro, or NSP5). These proteases are highly conserved

78 across coronavirus species and are absolutely required for viral replication ${ }^{17-20}$. Both proteases

79 are thus actively being investigated as targets for antivirals ${ }^{21}$. Recent Phase $2 / 3$ clinical trial

80 results with PF-07323332, a 3CL inhibitor, administered in combination with ritonavir, revealed

$8189 \%$ reduction in hospitalization with COVID-19 ${ }^{22}$. Both PLPro and 3CLPro are generated via

82 autocatalytic cleavage from the overlapping ORF1a and ORF1ab polyproteins, the first

83 translation products following SARS-CoV-2 infection. The ORF1a/ab polyproteins encode 16 
non-structural proteins (NSP1-16) that build the viral replication machinery (Fig 1A). The

nonstructural proteins are liberated from the ORF1a/ab polyprotein through cleavage by PLPro and 3CLPro, encoded by NSP3 and NSP5, respectively. Following this processing PLPro remains

87 bound to the endoplasmic reticulum membrane, while 3CLPro cleaves itself free, giving it access to the full cytosolic compartment. The impact of these proteases on the host proteome, and in particular the 3CLPro, remains poorly defined. Targeted screening of 300 interferonstimulated proteins in cell lines overexpressing SARS-CoV-2 3CLPro identified RNF20 as a target

91 of $3 \mathrm{CLpro}^{23}$. In a different screening of 71 immune pathway-related proteins, IRF3 was

92 identified as target of PLpro and NLRP12 and TAB1 as targets of 3CLpro, suggesting the role of

93 those proteases in the innate immune response to the virus ${ }^{24}$.

95 Only limited efforts have thus far been taken to identify systematically, and in unbiased fashion, 96 host cleavage targets of 3CLPro from SARS-CoV-2. Given the high conservation of 3CLPro, such

97 analysis would extend as well across coronavirus species. One approach taken recently used N-

98 terminomics to identify neo-N-termini generated by the viral proteases, and identified 14 new

99 cellular substrates ${ }^{25}$ and more than 100 substrates in a second study ${ }^{26}$. This approach is

100 limited, however, by the need for sufficient protein abundance and appropriate fragment size

101 and properties to be detected by mass spectrometry ${ }^{27,28}$. Only 3 cleavage targets have been

102 identified by more than one study to date (TAB1, ATAD2 and NUP107), reflecting the lack of

103 saturation of these approaches. Moreover, cleaved proteins that are subsequently degraded (a

104 process accelerated by infection) ${ }^{29,30}$ also escape detection by $\mathrm{N}$-terminomics, as do proteins

105 not expressed by the cell types used experimentally. In silico approaches provide the 
opportunity to overcome these numerous limitations, and to avoid laborious experimental screens. An initial such approach relied on similarity between cleavage sites in the viral polypeptide across divergent coronavirus species (NetCorona1.0) ${ }^{31}$. However, this method generates scores using assumptions about the viral cleavage site that do not apply to the SARS-

CoV-2 consensus sequence. For example, NetCorona1.0 predicts that a sequence containing a

111 proline at the $\mathrm{P} 2$ ' position can be cleaved, but this substitution has been shown to block

112 cleavage in cleavage assays ${ }^{32}$. In addition, NetCorona1.0 does not consider cleavage site

113 accessibility conferred by secondary structure, the relative efficiency of cleavage at different

114 sites, or the possibility that there may be host target sites of higher affinity than viral sites.

116 Here we combine published cleavage efficiency data on the SARS-CoV 3CLPro, which is $96 \%$

117 similar to SARS-CoV-2 3CLpro ${ }^{33}$, with genome-wide secondary structure analyses, to identify

118 and score 99,000+ predicted SARS-CoV/SARS-CoV-2 3CLPro cleavage sites across the human

119 proteome. Through score filtration and secondary structure analysis, we identify over 1000 high

120 likelihood sites. We re-discover nearly all prior SARS-CoV-2 3CLPro experimentally identified

121 sites, and we validate newly identified targets with purified reagents and in cell culture.

122 Focusing on cardiomyocyte-specific hits, we show 3CLPro leads to cleavage and degradation of

123 the sarcomeric protein obscurin (OBSCN) in human induced pluripotent cell-derived

124 cardiomyocytes (hiPSC-CM), and recapitulates the sarcomeric disorganization observed with

125 SARS-CoV-2 infection of hiPSC-CMs ${ }^{34-36}$. Our study provides a comprehensive atlas for

126 identifying the degradome of $3 \mathrm{CL}$ proteases, applicable to SARS-CoV-2 and, in light of the 
127 structural conservation of the $3 \mathrm{CL}$ protease across coronavirus species ${ }^{37}$, future coronavirus 128 outbreaks.

Bioinformatic prediction of SARS-CoV-2 3CLPro targets using experimental data from SARS-

133 We first sought to identify and score potential cleavage targets of the 3CLpro encoded by SARS-

134 COV-2. Given the $96 \%$ sequence similarity between 3CLPro from SARS-CoV-2 as well as the

135 homology in the viral genome cut sites ${ }^{17,33,37}$, we developed an algorithm based on

136 experimental data generated previously from SARS-CoV (2003) 3CLPro ${ }^{32}$. In this previous study,

137 FRET polypeptides spanning the first endogenous cut site between NSP4 and NSP5 (P5-

138 SAVLQSGF-P3') were generated and modified with every possible single amino acid substitution

139 from P5 to P3' position relative to the cleavage site. Cleavage efficiency by 3CLpro was then

140 assessed by fluorescence intensity compared to the consensus cleavage sequence. We

141 leveraged this data set to generate a score for every possible cleavage site using a lookup table,

142 multiplying the relative efficiency of each amino acid. This multiplication was then applied with

143 a sliding 8-amino acid windows across the entire human proteome (Fig 1B). Substitution at any

144 site that showed no detectable cleavage was interpreted as "0". Assuming a glutamine (Q) in

145 the P1 position, over 98,697 scored sites (>0) were identified. Expanding our search to include

146 methionine $(M)$ or histidine $(H)$ at P1 uncovered a total of 195,684 sites with a median score of

1470.0008 (Fig 1C) (Supplemental Table 1). GO analysis of scores in the top $15 \%(>0.01)$ showed 
148 an enrichment for cell-adhesion, morphogenesis and cytoskeletal genes (Supplemental Table

149 2). We named the algorithm Sarsport1.0.

151 To evaluate the precision of Sarsport1.0, we calculated scores for the 11 known 3CLPro cut sites

152 in the SARS-CoV viral genome. Scores ranged from $1.31-0.04$, all within the upper $5^{\text {th }}$

153 percentile of the score range. These scores were then compared with the published relative

$154 \mathrm{~K}_{\text {cat }} / \mathrm{K}_{\mathrm{m}}$ values for each cleavage site $^{38}$. With the exception of the cut-site between NSP9 and

155 NSP10 (ATVRLQ*AGNAT), our calculated score correlated closely with relative $\mathrm{K}_{\text {cat }} / \mathrm{K}_{\mathrm{m}}$ (Fig 1D

156 left). In contrast, there was essentially no correlation between NetCorona1.0 scores and

157 relative Kcat/Km (Fig 1D, right).

159 To evaluate the sensitivity of Sarsport1.0 to identify SARS-CoV-2 host protein targets, we next

160 calculated scores for the $>100$ recently published experimentally-identified SARS-CoV-2 3CLPro

161 cleavage targets. Sarsport1.0 identified 104 of 117 sites, including those with non-canonical

162 methionine or histidine at the P1 position (Fig 1C). The median score was over 0.1, which is

163 within the top $2.5 \%$ of all scores. Receiver operator curve (ROC) analysis (Fig 1E) showed

164 Sarsport1.0 to be highly predictive, with an area under the curve of 0.9473 , and $P<0.0001$. This

165 is likely an underestimate of true ROC, because true positives were likely missed in the

166 experimental approaches. We conclude that Sarsport1.0 is highly predictive of cleavage sites by

167 both SARS-CoV and SARS-CoV-2 3CLpro proteases. 
171 The unique high score but low $\mathrm{K}_{\mathrm{cat}} / \mathrm{K}_{\mathrm{m}}$ of the NSP9/10 cleavage site (Fig 1D) suggested that a

172 higher order structure, not captured by scoring based on primary sequence alone, might inhibit

173 cleavage. We therefore estimated the secondary structure of each cut site in the viral genome,

174 using the JPRED4 protein secondary structure prediction server ${ }^{39}$ and a 100aa window spanning

175 the P1 position. The NSP9/10 site in SARS-CoV was the only cleavage site where the P1 position

176 (Q) was predicted to lie in a $\beta$-sheet (Supplemental Table 3). In contrast, the other sites all lay

177 in predicted $\alpha$-helices or disordered regions, structures known to be more accessible to

178 proteases ${ }^{40,41}$. These data suggested that higher order structures such as $\beta$-sheets hinder

179 cleavage by 3CLpro.

181 To further probe this possibility, we used JPRED4 to evaluate secondary structures around all

182 predicted cleavage sites with a Q at P1 and with a Sarsport1.0 score $>0.1$ (4416 sites) (Fig 1F)

183 (Supplemental Table 4). The recent publication of predicted structures for most of the human

184 proteome with AlphaFold ${ }^{42}$ also provides the opportunity to cross validate secondary with

185 higher order structure. The relative frequency of $\beta$-sheet structures at the P1 position of

186 predicted cleavage sites was significantly less than the overall frequency of $\beta$-sheets for

187 glutamines in the proteome ${ }^{43}$ (Fig 1G), indicating that Sarsport1.0 partly biases away from $\beta$ -

188 sheets. Further comparison to published experimentally-identified cleavage sites revealed in

189 the latter an additional significantly increased propensity for cleavage in regions where P1 (Q) is

190 unstructured and in particular not in a $\beta$-sheet (Fig 1F). Thus, filtering results from Sarsport1.0

191 for the absence of a $\beta$-sheet structure at P1 will improve its positive predictive value. 
192 Interestingly, the median Sarsport1.0 score for sites that lie in unstructured regions (0.1024)

193 was significantly lower than for sites that lie in $\alpha$-helices $(0.1727)$ or $\beta$-sheets (0.27) (Fig 1G),

194 suggesting that the presence less permissive secondary structural order imposes a higher

195 evolutionary pressure for an optimal primary sequence cleavage motif.

196

Cleavage validation of novel targets

199 Because of the higher sensitivity of our method, we identified numerous new predicted

200 cleavage sites, in addition to those previously published. Gene Ontology (GO) analysis of

201 proteins with Sarsport1.0 score > 0.01 showed enrichment for many cell-adhesion proteins,

202 including many predicted cleavage sites located within homologous cadherin domains in the

203 cadherin protein superfamily (Supplemental Table 2). Evaluation with AlphaFold predicted

204 these sites to be in unstructured accessible loops within the cadherin domain, thus making

205 them likely to be cleaved if exposed to 3CLPro (Fig 1G). We validated these hits in vitro by

206 incubation of purified 3CLPro with commercially available recombinant cadherin proteins

207 (CDH6, CDH20), which have identical predicted sites (Score 0.145, Q203 and Q209, respectively,

208 Fig/Table XX). 3CLPro efficiently cleaved both CDH6 and CDH20, yielding the expected fragment

209 sizes based on the predicted cleavage site (Fig 2A). We similarly validated novel cleavage sites

210 in thrombin (IIA) and in the intracellular domain of NOTCH1: in vitro reactions with purified

211 proteins yielded expected fragment sizes for both (Fig 2B-D). The appearance of thrombin IIA

212 cleaved product was inhibited by the 3CLpro inhibitor GC376, demonstrating the requirement

213 of 3CLpro enzymatic activity (Fig 2B). Cleavage of NOTCH1 at a predicted site (Q2315, score 

0.432), within the intracellular domain of $\mathrm{NOTCH} 1$, yielded both predicted fragments (Fig $2 \mathrm{C}$ ).

215 Overexpression of 3CLPro in hiPSC-CMs also yielded NOTCH1 fragments of predicted length,

216 demonstrating cleavage in intact cells (Fig 2D). Additional targets chosen for their high score

217 and secondary structure accessibility (SVIL, UACA, NOTCH2) were similarly validated with

218 3CLPro overexpression in 293T cells, as was the previously published target TAB1

219 (Supplemental Fig 1A). Interestingly, in these cell overexpression experiments, while the levels

220 of full length target proteins were significantly reduced by expression of 3CLpro, the

221 appearance of fragments of predicted size were not observed. We hypothesized that cytosolic

222 fragments generated by 3CLPro may be further degraded by endogenous proteolytic pathways.

223 Supporting this notion, the plasma-membrane bound N-terminal cleavage product of full length

224 NOTCH1 yielded the expected 90kda fragment, while the C-terminal fragment only showed

225 reduction in total protein (Supplemental Fig 1B). We conclude that 3CLPro can cleave a wide

226 range of host proteins, and that the generated cytosolic protein fragments are likely often

227 degraded by endogenous pathways.

229 Cardiac targets of SARS-CoV-2 3CLPro show multiple cut sites across sarcomeric proteins

230 Previous work has demonstrated disorganization of sarcomeres after SARS-CoV-2 infection of

231 hiPSC-derived cardiomyocytes ${ }^{34-36,44}$. We hypothesized that 3CLPro may be degrading

232 sarcomere proteins directly. Consistent with this notion, overexpression of 3CLPro, but not a

233 catalytically inactive mutant (C145A), in hiPSC-CMs led to pronounced sarcomere breakdown

234 within $48 \mathrm{~h}$ (Fig $3 \mathrm{~A}$ ). At this $48 \mathrm{~h}$ time point, we also observed numerous cells with a

235 stereotypical intermediate phenotype, in which sarcomeres exhibited increased length, as 
236 defined by the distance between alpha-actinin stained Z-discs (Fig 3B-C), suggesting that a key

237 structural protein of the sarcomere is being targeted by 3CLpro.

239 We applied our in silico primary and secondary analysis to identify putative sarcomere targets

240 of 3CLpro (Supplemental Table 5). Within this list, we identified the giant protein Obscurin

241 (OBSCN) as a probable target, with 5 high-likelihood sites along the length of the 800kda

242 (>7500 amino acids) protein (Fig 4A). Consistent with these data, hiPSC CMs expressing 3CLpro

243 had a marked reduction in Obscurin protein, compared to cells expressing the C145A mutant

244 (Fig4B-C). The reduction was apparent using antibodies against multiple epitopes along this

245 large protein. In contrast, levels of alpha-actinin (ACTN2) and myosin heavy chain (MYH6),

246 which our algorithm did not predict to be cleaved by 3CLpro, were not altered (Fig 4B). In

247 addition, immunocytochemistry showed loss of Obscurin in otherwise apparently intact, alpha-

248 actinin-positive sarcomeres in cells expressing 3CLPro, but not cells expressing C145A (Fig 4C).

250 As with a number of targets described above (Supplemental Fig 1) we did not detect any

251 Obscurin fragments, despite using antibodies that recognize multiple epitopes along the length

252 of the protein. To test whether the absence of fragments might be due to endogenous

253 proteosome activity, we treated cardiomyocytes expressing 3CLPro, versus C145A, with the

254 proteosome inhibitor MG132, and collected cellular protein 24h later. Blotting with the two

255 antibodies that recognize the region between cut sites 3 and 4 (Q4075 and Q5488, respectively)

256 yielded the expected fragment size ( 155kda) (Fig 4D), validating the predicted sites as a

257 3CLpro targets, and demonstrating that the ensuing fragment is targeted for degradation by the 
258 proteosome. Identification of other fragments within Obscurin was technically unfeasible due

259 to either lack of antibodies against the specific region, or to overlap with non-specific bands on

260 blots. However, we also observed the appearance, after proteasome inhibition, of fragments of

261 predicted size in Supervillin (SVIL), another giant sarcomeric protein predicted to be targeted by

262 our algorithm, as well as TAB1, which was previously shown to be targeted by 3CLpro but for

263 which no fragments had been detected ${ }^{24}$

Obscurin degradation in SARS-CoV-2 infection

266 Finally, we tested if those results were recapitulated in a model of hPSC-CMs infected with live

267 SARS-CoV-2. For these experiments, we used two hPSC-CM lines, WTC-11c (hiPSC-CMs) and H7

268 (hESC-CMs, human embryonic stem cell-derived cardiomyocytes), previously used to study the

269 effects of SARS-CoV-2 on human cardiomyocytes ${ }^{35}$. Within 48 h of infection, coincident with the

270 robust appearance of viral nucleocapsid, total Obscurin protein gradually reduced by $40-60 \%$

271 (Fig 5A-B). In contrast, protein abundance of ACTN2, MYH6, and MYH7 was unaffected by

272 infection (Fig 5B), mirroring the effects seen with 3CLpro alone (Fig 4B). Similarly,

273 immunocytochemistry of infected cells showed reduction of Obscurin staining in cells

274 expressing viral nucleocapsid, despite seemingly intact sarcomeres (TNNT2 staining), again

275 mirroring the effects seen with 3CLpro alone (Fig 4D). Thus the loss of Obscurin caused by

276 3CLpro-mediated cleavage might explain the loss of sarcomere integrity during SARS-CoV-2

277 infection in human cardiomyocytes.

278

279

Discussion 
280 We leveraged here experimental data and genome-wide secondary structure analyses to

281 develop a reliable computational algorithm, Sarsport1.0, that predicts endogenous non-viral

282 cleavage targets by the 3CLpro SARS-CoV-2 protease across the human proteome. We validated

283 the precision of the algorithm by confirming novel predicted cleavage sites, using both

284 biochemical and cell culture approaches. The algorithm is specific: all 8 predicted host proteins

285 that we chose to test experimentally confirmed cleavage by 3CLpro. The algorithm is also

286 precise, accurately correlating scores with the known $\mathrm{K}_{\mathrm{m}} / \mathrm{K}_{\text {cat }}$ values for the cut sites in the

287 single viral polypeptide. The single exception to this correlation, the NSP9/10 site, is also the

288 only site lying within a predicted beta-sheet, which likely hinders protease access. The

289 particularly high score of the NSP9/10 site (score $=1.31$ ) may have evolved to overcome this

290 more inaccessible higher order structure.

291

292 Finally, the algorithm is highly sensitive, accurately predicting high cut scores in nearly all

293 previously experimentally identified 3 CLpro sites ${ }^{23-26}$. In addition, thousands of novel sites are

294 predicted to be cleaved by 3CLpro. Numerous reasons likely explain why the computational

295 algorithm is more sensitive than prior experimental approaches: (1) experimental paradigms

296 are limited to detecting the proteins expressed in the chosen experimental cells; (2) proteomic

297 approaches rely on the ability to detect new protein fragments by mass spectrometry, a

298 relatively insensitive method; (3) and proteomic approaches also require the physical presence

299 of cleaved products, but as shown here, these fragments are often quickly degraded after

300 cleavage. Our computational algorithm overcomes these limitations and provides a

301 comprehensive atlas of the putative SARS-CoV-2 3CLpro degradome. 
303 The SARS-CoV-2 3CLpro is the target of ongoing therapeutic efforts to treat COVID-19. Recent 304 interim analysis of the phase 2/3 EPIC-HR (Evaluation of Protease Inhibition for COVID-19 in 305 High-Risk Patients) ${ }^{22}$, which tested the combination therapy of PF-07321332, a 3CLpro inhibitor, 306 with ritonavir, a CYP3A4 inhibitor that prevents the metabolism of protease inhibitors, reported 307 a nearly $90 \%$ reduction in hospitalization or death compared to placebo in non-hospitalized 308 high-risk adults with COVID-19 ( $<<0.0001)$. Our data suggest that these remarkable benefits of 3093 CLpro inhibition may accrue from effects beyond suppression of viral replication. For example, 310 infected cells that do not sustain replication may nevertheless experience significant cellular

311 damage from 3CLpro activity on the host proteome, and PF-07321332 and other 3CLpro

312 inhibitors would be predicted to prevent this cellular damage. Similarly, expression of 3CLpro is

313 one of the earliest events in the viral life cycle, and may thus cause early cellular damage,

314 potentially suppressing cellular defenses against the ensuing viral replication. Lingering effects

315 of 3CLpro may also contribute to persistent symptoms, as observed with the long-COVID

316 syndrome. In sum, the remarkable benefits of 3CLpro inhibition in COVID-19 patients

317 underscores the need to further understand the impact of 3CLpro on the host proteome, which 318 will be substantially aided by our predictive algorithm.

320 To validate our algorithm, we investigated the effects of 3CLPro in two different models (OE

321 and live SARS-CoV-2) on hiPSC-CMs, previously shown to be susceptible to SARS-CoV-2

322 infection with profound effects on sarcomeric organization ${ }^{34-36}$. We identified the giant

323 sarcomeric protein Obscurin as a target of 3CLpro, and showed (1) that both ectopic expression 
324 of 3CLpro and infection by SARS-CoV-2 cleave and degrade Obscurin, while leaving other

325 sarcomeric proteins intact; and (2) that ectopic expression of 3CLpro, but not a mutant without

326 enzymatic activity, causes sarcomeric disorganization in a stereotypical fashion similar to that

327 observed with SARS-COV-2 infection, and consistent with the pattern predicted by degradation

328 of Obscurin, an important structural component of the Z-disk. Thus, we propose that

329 sarcomeric disorganization during SARS-CoV-2 infection is likely caused in large part by direct

330 proteolysis of Obscurin by 3CLpro.

332 Cardiac complications of COVID19 have been well documented, and the presence of cardiac

333 damage, as reflected in plasma troponin levels, is highly predicted of morbidity and mortality

334 after SARS-COV-2 infection ${ }^{45-47}$. It is still unclear if the pathology observed in the heart is due to

335 a direct cytotoxic effect of the virus or secondary to the systemic inflammation. Some evidence

336 of direct infection by SARS-CoV-2 in the human heart has been reported, suggesting that a

337 direct effect is possible ${ }^{48,49}$, although other post-mortem studies have not detected infected

338 cardiomyocytes in COVID-19 patients ${ }^{44-46}$. Thus, the implications of our findings in iPSCMs on

339 human cardiac disease should be interpreted cautiously. We used these studies primarily as

340 molecular and structural validation of our predictive algorithm. The use of hPSC-CMs also offers

341 a powerful tool for pharmacological and drug screening, in addition to disease modeling.

343 In summary, we provide a validated, computationally derived, comprehensive atlas of the

344 putative 3CLpro degradome, overcoming limitations in sensitivity inherent to experimental

345 approaches. Our findings provide a powerful tool to aid investigations into the virus-host 
346 interactions mediated by 3CLpro, the target of highly efficacious therapy against COVID-19. In

347 light of the structural conservation of the $3 C L$ protease across coronavirus species, such

348 investigations will likely also apply to future coronavirus outbreaks.

\section{Acknowledgements}

351 ZA was supported by NIH/NHLBI (HL152446). NY was supported by NIH training grant

352 5T32AR053461. Q.M was supported by NIAMS T32 training grant (AR 53461-12). SM was

353 supported by post-doctoral fellowship from the Institute for Stem Cells and Regenerative

354 Medicine (University of Washington). This work was also supported by R01 HL128362 and R01

355 HL146868 to C.E.M.; the Robert B. McMillen Foundation and the State of Washington

356 philanthropical support to the UW Institute for Stem Cell and Regenerative Medicine. We

357 would also like to acknowledge the staff of the University of Washington BSL-3 facility, as well

358 as our Environmental Health and Safety staff, who provided assistance during experiments and

359 ensured our safety.

\section{Conflicts of interest}

363 The authors declare no conflicts 


\section{Works Cited}

1. Dong, E., Du, H. \& Gardner, L. An interactive web-based dashboard to track COVID-19 in real time. Lancet Infect. Dis. 20, 533-534 (2020).

2. Polak, S. B., Van Gool, I. C., Cohen, D., von der Thüsen, J. H. \& van Paassen, J. A systematic review of pathological findings in COVID-19: a pathophysiological timeline and possible mechanisms of disease progression. Mod. Pathol. 33, 2128-2138 (2020).

3. Guan, W. et al. Clinical Characteristics of Coronavirus Disease 2019 in China. N. Engl. J. Med. 382, 1708-1720 (2020).

4. Wiersinga, W. J., Rhodes, A., Cheng, A. C., Peacock, S. J. \& Prescott, H. C. Pathophysiology, Transmission, Diagnosis, and Treatment of Coronavirus Disease 2019 (COVID-19): A Review. JAMA 324, 782-793 (2020).

5. AlSamman, M., Caggiula, A., Ganguli, S., Misak, M. \& Pourmand, A. Non-respiratory presentations of COVID-19, a clinical review. Am. J. Emerg. Med. 38, 2444-2454 (2020).

6. Davis, H. E. et al. Characterizing long COVID in an international cohort: 7 months of symptoms and their impact. EClinicalMedicine 38, 101019 (2021).

7. Nasserie, T., Hittle, M. \& Goodman, S. N. Assessment of the Frequency and Variety of Persistent Symptoms Among Patients With COVID-19: A Systematic Review. JAMA Netw. Open 4, e2111417-e2111417 (2021).

8. Gordon, D. E. et al. A SARS-CoV-2 protein interaction map reveals targets for drug repurposing. Nature 583, 459-468 (2020).

9. Wang, R. et al. Genetic Screens Identify Host Factors for SARS-CoV-2 and Common Cold Coronaviruses. Cell 184, 106-119.e14 (2021).

10. Schneider, W. M. et al. Genome-Scale Identification of SARS-CoV-2 and Pan-coronavirus Host Factor Networks. Cell 184, 120-132.e14 (2021).

11. Badorff, C. et al. Enteroviral protease $2 A$ cleaves dystrophin: evidence of cytoskeletal disruption in an acquired cardiomyopathy. Nat. Med. 5, 320-326 (1999).

12. Lim, B.-K. et al. Inhibition of Coxsackievirus-associated dystrophin cleavage prevents cardiomyopathy. J. Clin. Invest. 123, 5146-5151 (2013).

13. Robinson, K. S. et al. Enteroviral 3 C protease activates the human NLRP1 inflammasome in airway epithelia. Science 370, (2020).

14. Nie, Z. et al. Human immunodeficiency virus type 1 protease cleaves procaspase 8 in vivo. J. Virol. 81, 6947-6956 (2007).

15. Strack, P. R. et al. Apoptosis mediated by HIV protease is preceded by cleavage of Bcl-2. Proc. Natl. Acad. Sci. U. S. A. 93, 9571-9576 (1996).

16. Morazzani, E. M. et al. Proteolytic cleavage of host proteins by the Group IV viral proteases of Venezuelan equine encephalitis virus and Zika virus. Antiviral Res. 164, 106122 (2019).

17. Hegyi, A. \& Ziebuhr, J. Conservation of substrate specificities among coronavirus main proteases. J. Gen. Virol. 83, 595-599 (2002).

18. MacDonald, E. A. et al. Recognition of Divergent Viral Substrates by the SARS-CoV-2 Main Protease. ACS Infect. Dis. 7, 2591-2595 (2021).

19. Shitrit, A. et al. Conserved interactions required for inhibition of the main protease of severe acute respiratory syndrome coronavirus 2 (SARS-CoV-2). Sci. Rep. 10, 20808 
(2020).

20. V'kovski, P., Kratzel, A., Steiner, S., Stalder, H. \& Thiel, V. Coronavirus biology and replication: implications for SARS-CoV-2. Nat. Rev. Microbiol. (2020). doi:10.1038/s41579-020-00468-6

21. Osipiuk, J. et al. Structure of papain-like protease from SARS-CoV-2 and its complexes with non-covalent inhibitors. Nat. Commun. 12, 743 (2021).

22. Boras, B. et al. Preclinical characterization of an intravenous coronavirus 3CL protease inhibitor for the potential treatment of COVID19. Nat. Commun. 12, 6055 (2021).

23. Zhang, S., Wang, J. \& Cheng, G. Protease cleavage of RNF20 facilitates coronavirus replication via stabilization of SREBP1. Proc Natl Acad Sci USA 118, e2107108118 (2021).

24. Moustaqil, M. et al. SARS-CoV-2 proteases PLpro and 3CLpro cleave IRF3 and critical modulators of inflammatory pathways (NLRP12 and TAB1): implications for disease presentation across species. Emerg. Microbes Infect. 10, 178-195 (2021).

25. Meyer, B. et al. Characterising proteolysis during SARS-CoV-2 infection identifies viral cleavage sites and cellular targets with therapeutic potential. Nat. Commun. 12, 5553 (2021).

26. Pablos, I. et al. Mechanistic insights into COVID-19 by global analysis of the SARS-CoV-2 3CL ${ }^{\text {pro }}$ substrate degradome. Cell Rep. 37, (2021).

27. Kaushal, P. \& Lee, C. N-terminomics - its past and recent advancements. J. Proteomics 233, 104089 (2021).

28. Angel, T. E. et al. Mass spectrometry-based proteomics: existing capabilities and future directions. Chem. Soc. Rev. 41, 3912-3928 (2012).

29. Valerdi, K. M., Hage, A., van Tol, S., Rajsbaum, R. \& Giraldo, M. I. The Role of the Host Ubiquitin System in Promoting Replication of Emergent Viruses. Viruses 13, (2021).

30. Tran, K., Mahr, J. A. \& Spector, D. H. Proteasome subunits relocalize during human cytomegalovirus infection, and proteasome activity is necessary for efficient viral gene transcription. J. Virol. 84, 3079-3093 (2010).

31. Kiemer, L., Lund, O., Brunak, S. \& Blom, N. Coronavirus 3CLpro proteinase cleavage sites: possible relevance to SARS virus pathology. BMC Bioinformatics 5, 72 (2004).

32. Chuck, C.-P. et al. Profiling of Substrate Specificity of SARS-CoV 3CLpro. PLoS One 5, e13197 (2010).

33. Zhang, L. et al. Crystal structure of SARS-CoV-2 main protease provides a basis for design of improved $\alpha$-ketoamide inhibitors. Science 368, 409-412 (2020).

34. Perez-Bermejo, J. A. et al. SARS-CoV-2 infection of human iPSC-derived cardiac cells reflects cytopathic features in hearts of patients with COVID-19. Sci. Transl. Med. 13, (2021).

35. Marchiano, S. et al. SARS-CoV-2 Infects Human Pluripotent Stem Cell-Derived Cardiomyocytes, Impairing Electrical and Mechanical Function. Stem cell reports 16, 478-492 (2021).

36. Li, Y. et al. SARS-CoV-2 induces double-stranded RNA-mediated innate immune responses in respiratory epithelial-derived cells and cardiomyocytes. Proc. Natl. Acad. Sci. 118, e2022643118 (2021). structural and functional features of coronavirus protease nsp5 (3CLpro, Mpro) in the 
age of COVID-19. J. Gen. Virol. 102, (2021).

38. Grum-Tokars, V., Ratia, K., Begaye, A., Baker, S. C. \& Mesecar, A. D. Evaluating the 3C-like protease activity of SARS-Coronavirus: recommendations for standardized assays for drug discovery. Virus Res. 133, 63-73 (2008).

39. Drozdetskiy, A., Cole, C., Procter, J. \& Barton, G. J. JPred4: a protein secondary structure prediction server. Nucleic Acids Res. 43, W389-94 (2015).

40. Li, F. et al. Procleave: Predicting Protease-specific Substrate Cleavage Sites by Combining Sequence and Structural Information. Genomics. Proteomics Bioinformatics 18, 52-64 (2020).

41. Timmer, J. C. et al. Structural and kinetic determinants of protease substrates. Nat. Struct. Mol. Biol. 16, 1101-1108 (2009).

42. Jumper, J. et al. Highly accurate protein structure prediction with AlphaFold. Nature 596, 583-589 (2021).

43. Chou, P. Y. \& Fasman, G. D. Conformational parameters for amino acids in helical, $\beta$ sheet, and random coil regions calculated from proteins. Biochemistry 13, 211-222 (1974).

44. Bailey, A. L. et al. SARS-CoV-2 Infects Human Engineered Heart Tissues and Models COVID-19 Myocarditis. JACC. Basic to Transl. Sci. 6, 331-345 (2021).

45. Majure, D. T. et al. Usefulness of Elevated Troponin to Predict Death in Patients With COVID-19 and Myocardial Injury. Am. J. Cardiol. 138, 100-106 (2021).

46. García de Guadiana-Romualdo, L. et al. Cardiac troponin and COVID-19 severity: Results from BIOCOVID study. Eur. J. Clin. Invest. 51, e13532 (2021).

47. Lombardi, C. M. et al. Association of Troponin Levels With Mortality in Italian Patients Hospitalized With Coronavirus Disease 2019: Results of a Multicenter Study. JAMA Cardiol. 5, 1274-1280 (2020).

48. Pesaresi, M. et al. SARS-CoV-2 identification in lungs, heart and kidney specimens by transmission and scanning electron microscopy. Eur. Rev. Med. Pharmacol. Sci. 24, 5186-5188 (2020). MEDIATED CARDIOMYOCYTE FUSION MAY CONTRIBUTE TO INCREASED ARRHYTHMIC RISK IN COVID-19. Hear. Rhythm 18, S35-S35 (2021). 
A.
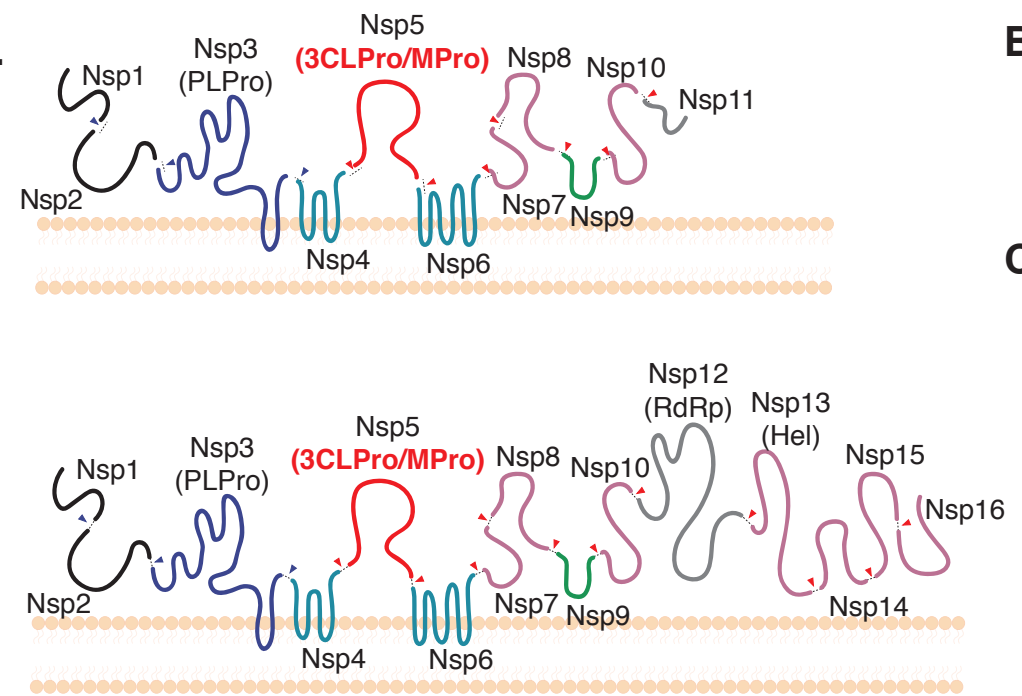

D.

Predicted score vs Relative Kcat/Km

(SARS-COV 3CLPro sites)
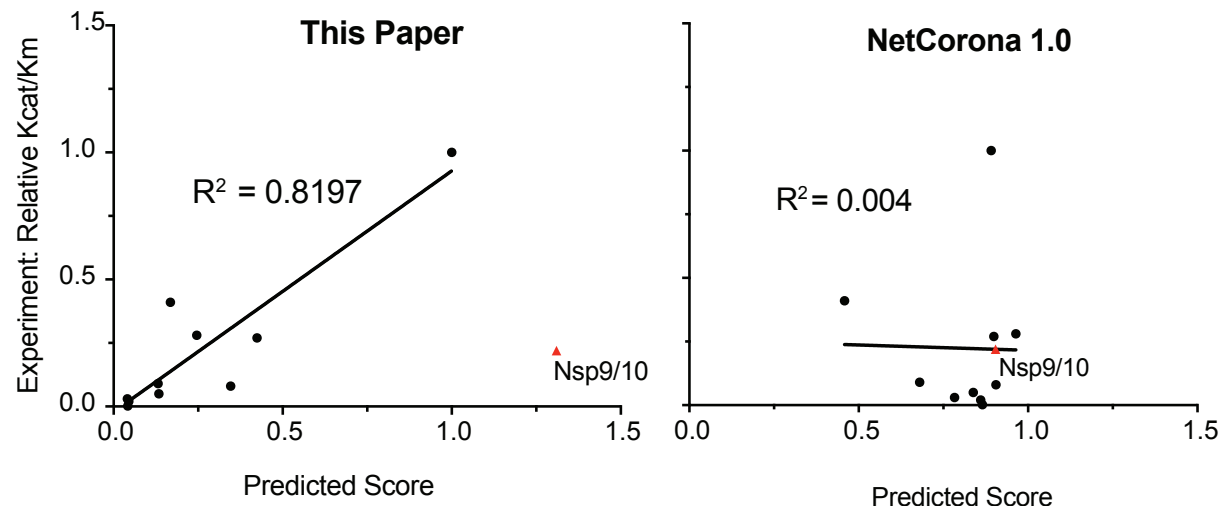

F.

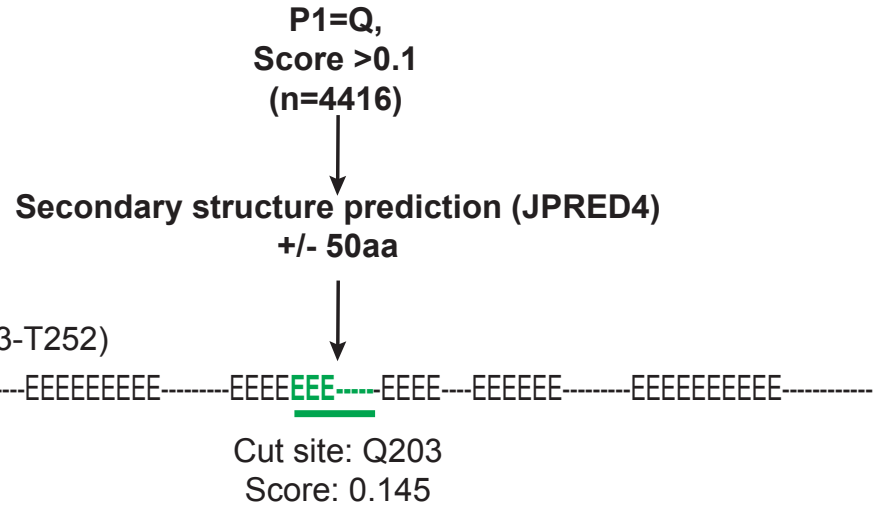

Structure validation (Alpha-fold)

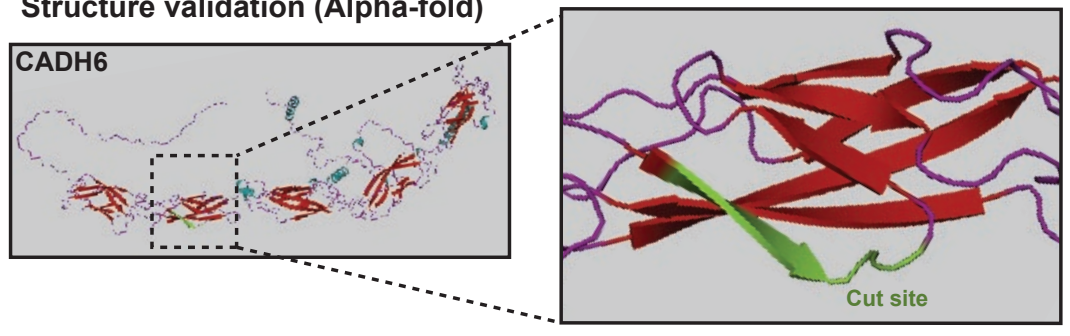

C.

Identify 8-amino acid window Multiply scores at each position
centered around $\mathrm{P} 1(\mathrm{Q} / \mathrm{M} / \mathrm{H})$

$\mathrm{P} 5$ P4 P3 P2 P1 P1' P2' P3' $\rightarrow$ P5 *P4 *P3 *P2 *P1*P1' *P2' *P3'<smiles>[AlH2]</smiles>

195,684 scored sites

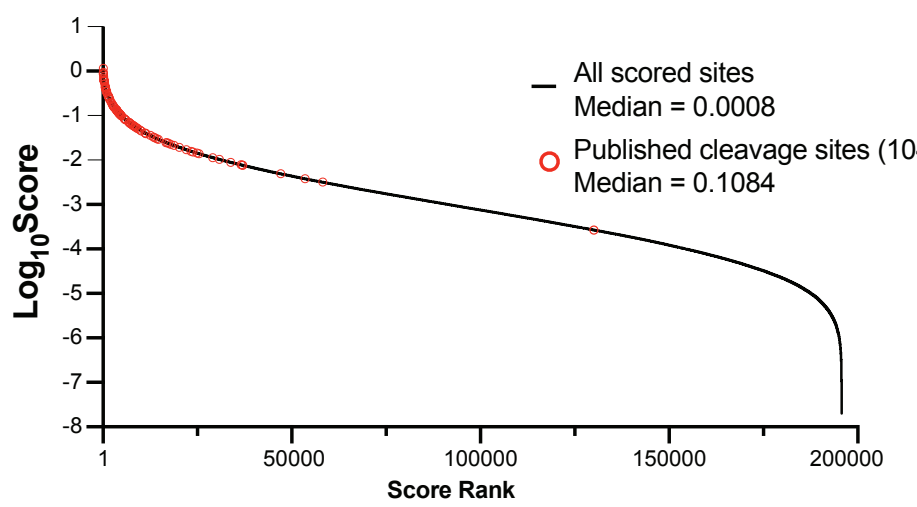

E.

ROC

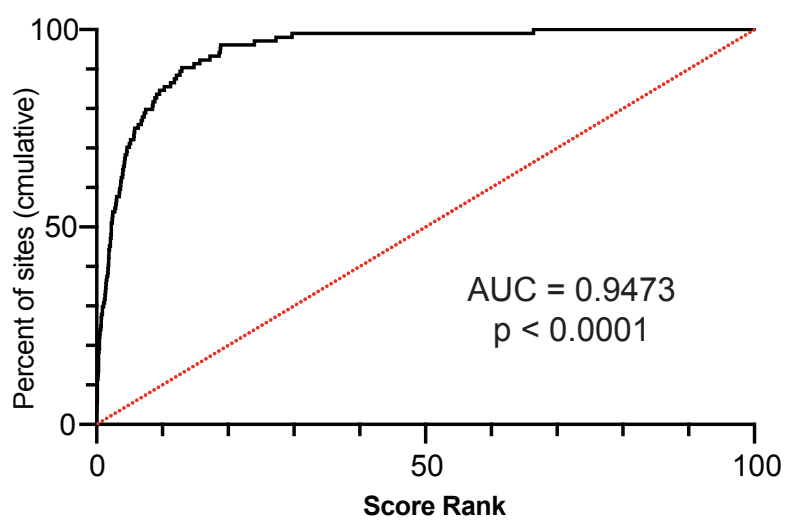

G.

Secondary structure $\mathbf{P} 1=\mathbf{Q}$

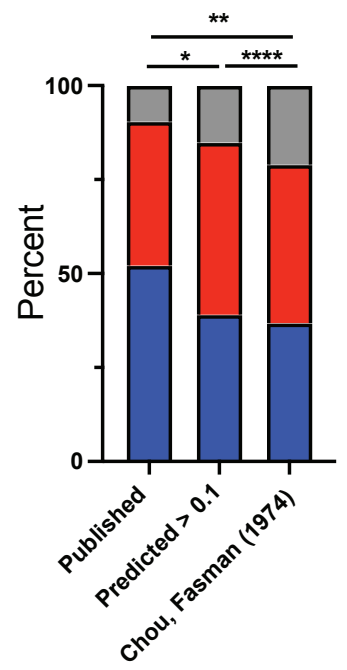

$\square$ Disordered (-)

$\square$ Helix $(\mathrm{H})$

$\square$ Beta-Sheet (B)
G. Score distribution
$\mathrm{P} 1=\mathrm{Q}$

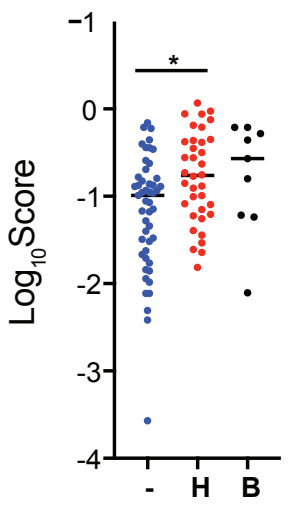

Secondary at $\mathrm{P} 1(\mathrm{Q})$ 
Figure 1

A) Diagram of endogenous function of the SARS-CoV-2 3C-like protease (3CLPro). 3CLPro cleaves at 11 sites within the 2 large polypeptides pp1a and pp1ab generated from the overlapping reading frames ORF1a and ORF1ab (respectively). Cleavage by 3CLPro, as well as the other viral protease papain-like protease (PLPro) liberates the nonstructural proteins (NSPs) that are required for viral transcription, replication, suppression of host immune responses and suppression of host gene expression.

B) Work-flow for bioinformatic identification and scoring of putative 3CLPro cleavage sites withing the human proteome. Scores for each position along the cleavage site (P5-P3') were obtained from experimental data from SARS-CoV 3CLPro (Chuck, et al 2010). First $P 1$ positions were identified $(\mathrm{Q}, \mathrm{M}$ or $\mathrm{H})$, and overall cleavage score generated by multiplying scores for each amino acid around P1. Within each 8-amino acid window, any position that contained an amino acid that scored a "ND" (no cleavage detected) in Chuck, et al resulted in a score of 0 . Overall, 195,684 scored cleavage sites ( $>0)$ were detected across the human proteome.

C) Distribution of all scores ( $\log _{10} \mathrm{Score}$ ). Scores of published cleavage sites detected by our prediction are highlighted in red.

D) Correlation of predicted score with experimentally-derived $K_{\text {cat }} / K_{m}$ values for SARS-COV 3CLPro (Grums-Tokars, 2008). Scores generated in this study are shown on the left, and scores generated by NetCorona1.0 (Kiemer, 2004). Shown on the right. For $\mathrm{R}^{2}$ calculations, the cleavage site between NSP9 and NSP10 for both graphs.

E) Receiver operator curve analysis to assess predictive power of bioinformatic scoring based on scores of published cleavage sites. Cumulative percentage of scores captured plotted vs score rank (highest score $=1$ to lowest score $=100$ ), and area under the curve (AUC) captured. 95\% confidence interval determined by Wilson/Brown method.

F) Secondary structure analysis of high scoring sites $(>0.1)$ with $P 1=Q$. To increase secondary structure accuracy, a 100aa window centered around P1 (Q) was identified. Resulting 100aa peptides were analyses by JPRED4 to predict secondary structure ( " " = unstructured, " $H$ " = alpha-helix, "E" = beta-sheet). When available, candidate cleavage sites were verified by alpha-Fold structure. Highlighted is a predicted site in Cadherin- 6 (CADH6).

G) Fraction of each $P 1(Q)$ that lies in each type of secondary structure (unstructured, alpha-helix, beta sheet). Comparisons shown for predicted cleavage sites with score > 0.1 vs published cleavage sites (all scores) vs published secondary structure distribution of all glutamines (Q). Statistical analysis of secondary structure distribution calculated using Chi-squared goodness of fit.

H) Score distribution of published cleavage sites $(P 1=Q)$, striated by secondary structure. Statistical analysis calculated by one-way ANOVA with Holm-Sidak multiple comparison test.

525

For all statistics shown, ${ }^{*}=p \leq 0.05,{ }^{* *} p \leq 0.01,{ }^{* * *} p \leq 0.001,{ }^{* * * *}=p \leq 0.00001$ 
Fig 2.

A. Recombinant Cadherin (extracellular domain)

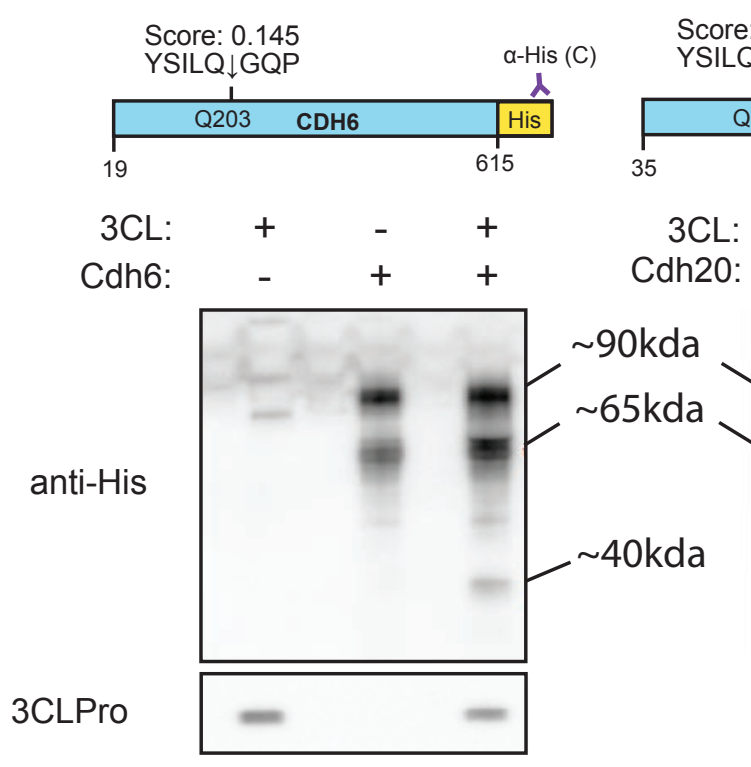

C. Recombinant $\mathrm{NOTCH} 1$ (His-2280-2550)
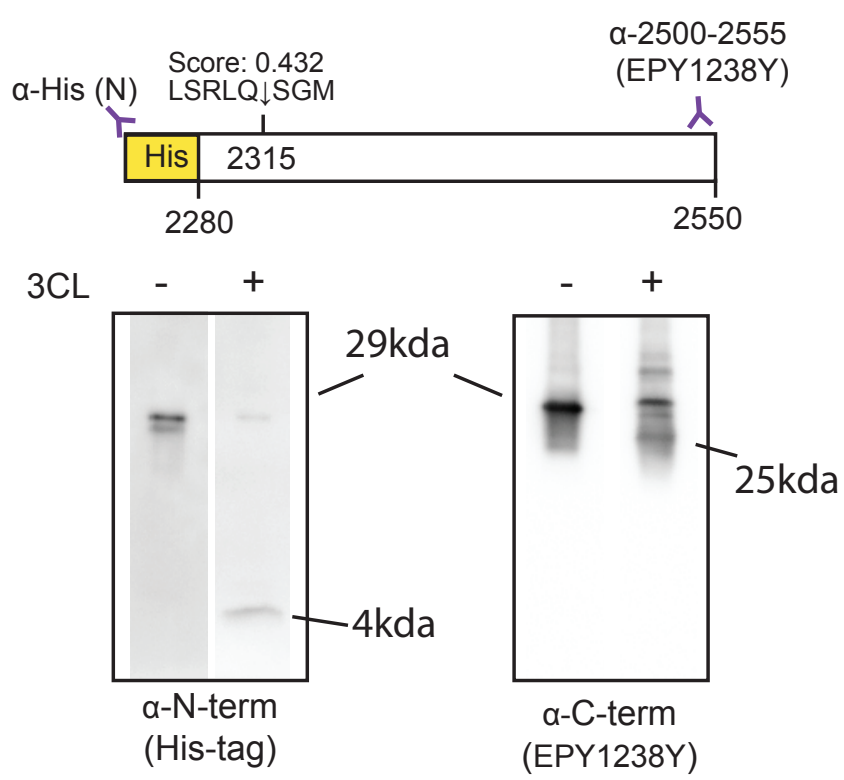

B.

Ila

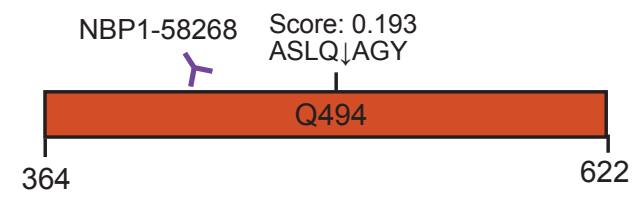

$\begin{array}{rlllll}\text { Ila: } & - & - & + & + & + \\ \text { 3CL: } & + & + & - & + & + \\ \text { GC376: } & - & + & - & - & +\end{array}$

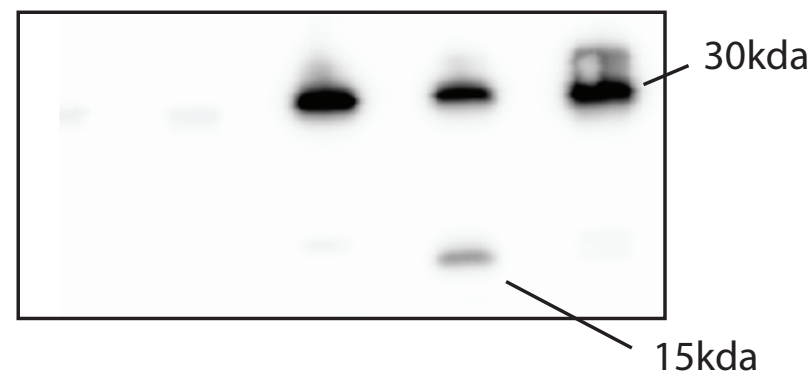

D.

Endogenous NOTCH1

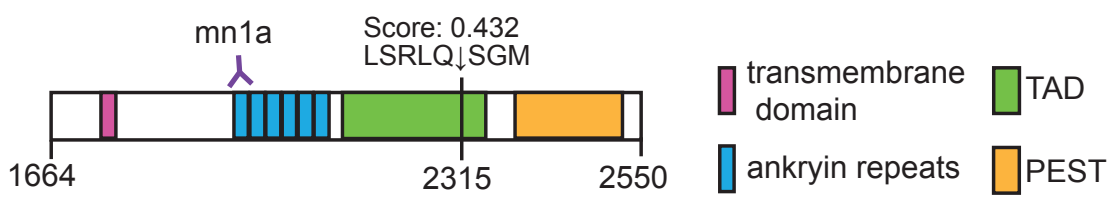

1664

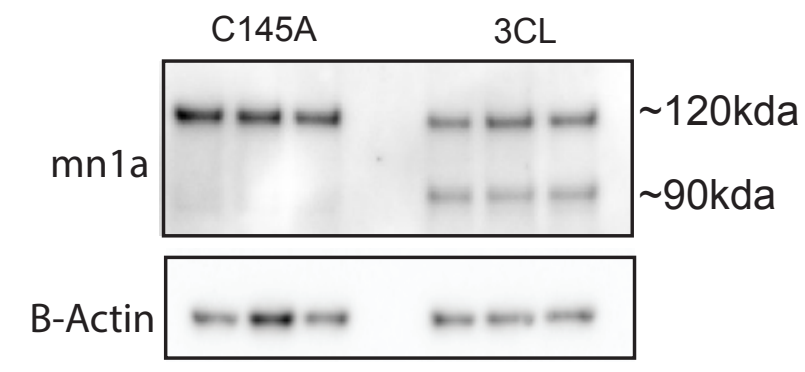


Figure 2

A) Western blot of in vitro cleavage of recombinant cadherin with purified 3CLPro. 1uM of purified 3CLPro was incubated with 2ug of recombinant $\mathrm{CDH} 6$ or $\mathrm{CDH} 20$ (C-terminal HisTag) in a 50uL reaction for $1 \mathrm{hr}$. Shown are cleavage sites within the recombinant fragment, with amino acid positions displayed for the full length proteins. Western blots showing staining against the C-terminus (His-Tag) of each protein and 3CLPro. Recombinant proteins are a mixture of glycosylated $(\sim 90 \mathrm{kDa})$ and unglycosylated ( $\sim 5 \mathrm{kda})$, corresponding to cleavage fragments of $\sim 62 \mathrm{kDa}$ and $40 \mathrm{kDa}$ (respectively).

B) Western blot of in vitro cleavage of purified human alpha thrombin (Ila). Diagram shows amino acid position of unprocessed prothrombin. Position of cleavage site shown with respect to epitope of antibody used for western blot. 1uM of purified 3CLpro was incubated with 2 ug alpha thrombin overnight under reducing conditions, with or without the 3CLPro inhibitor GC376 (1uM).

C) In vitro cleavage of purified recombinant NOTCH1 fragment (aa2280-2550) with a Nterminal His-Tag. Reactions were done with $1 \mathrm{uM}$ of purified 3CLPro for $1 \mathrm{hr}$. Diagram shows position of cleavage within the NOTCH1 fragment, with amino acid positions corresponding to the full length protein. Epitope regions showed for antibody with epitope C-terminal to the cleavage site. Full length size is $\sim 29 \mathrm{kDa}$, with $\mathrm{N}$ and C-terminal fragments of $4 \mathrm{kDa}$ and $25 \mathrm{kDa}$ (respectively).

550

551

D) Cellular cleavage of NOTCH1. Western blots show lysates of hIPSC cardiomyocytes expressing 3CLPro or catalytically inactive C145A variant for 48h. Cleavage site position within the intracellular fragment of NOTCH1 shown, as well as epitope for antibody used in western blot. 


\section{Fig 3}

A.

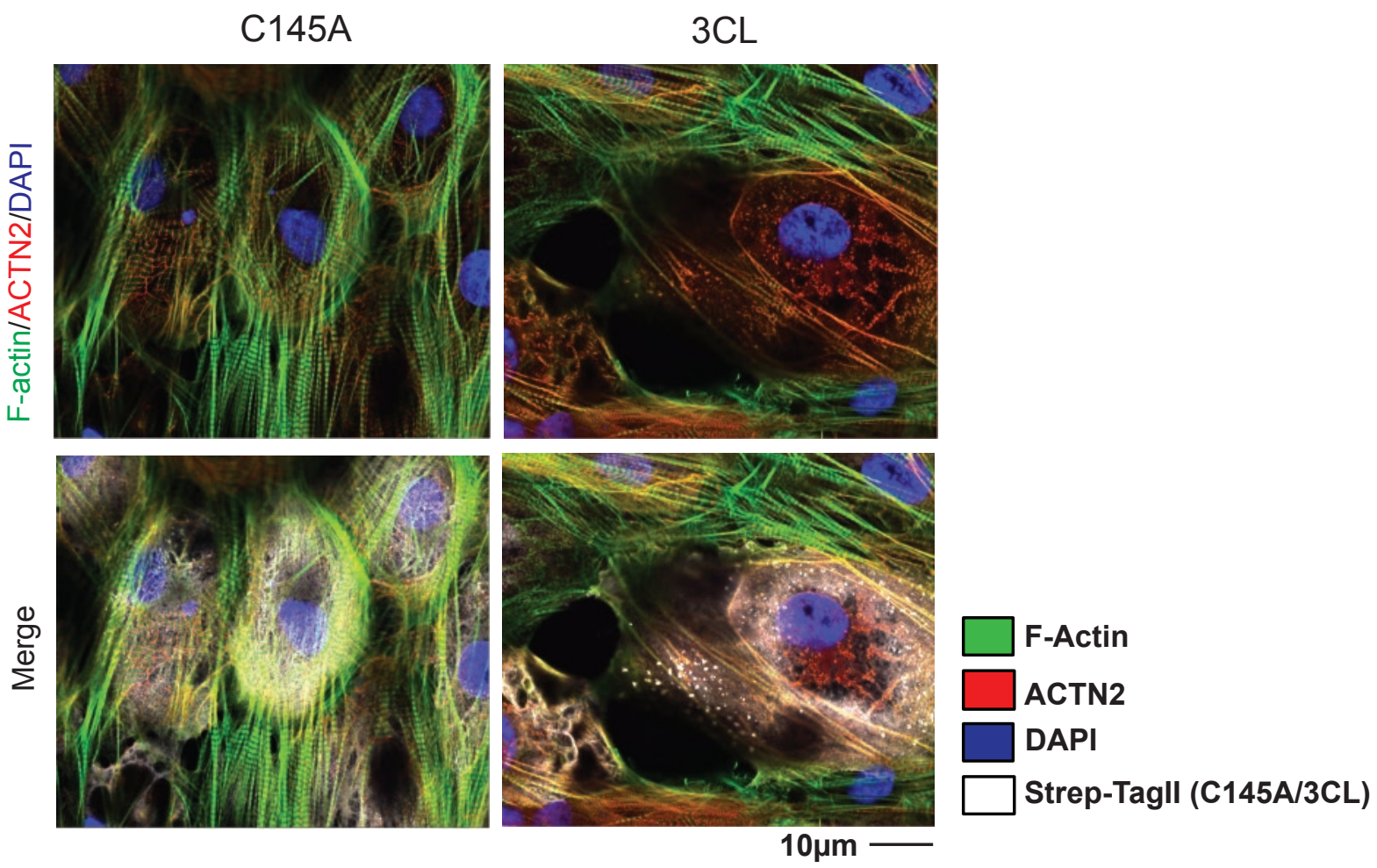

C145A

B.
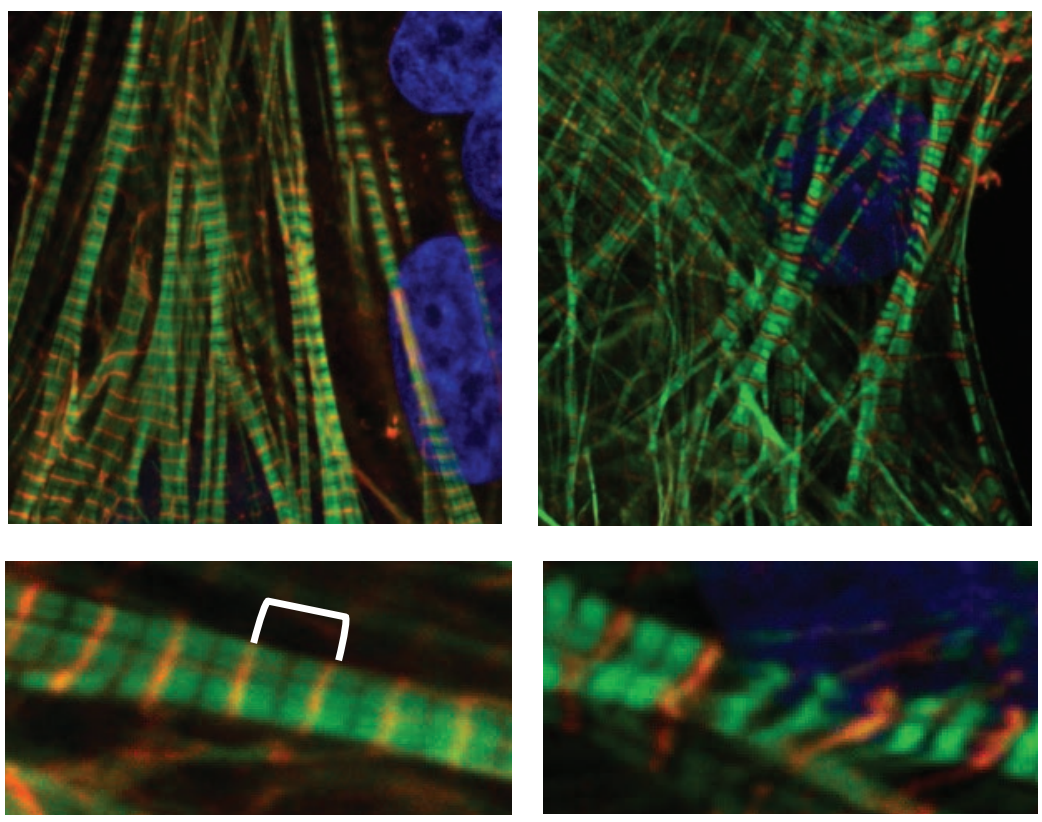

3CL

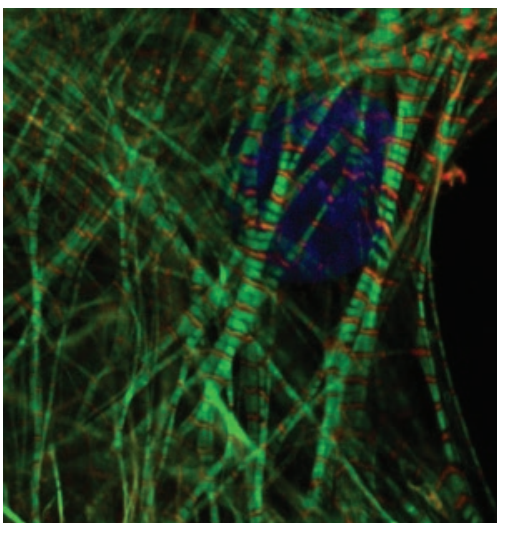

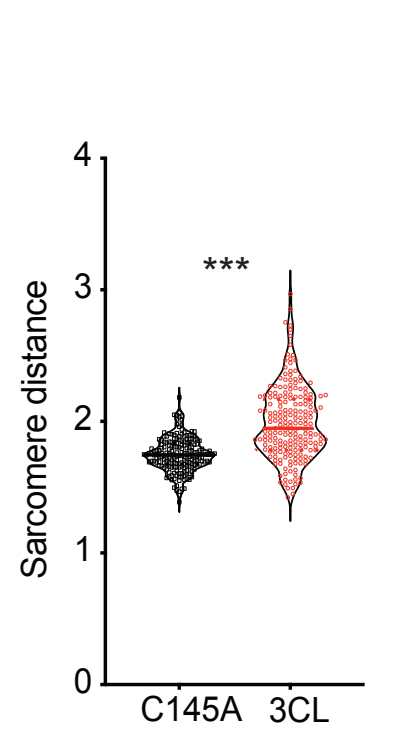

C.
F-Actin

ACTN2

DAPI 
552 Figure 3

553

554

555

556

557

558

559

560

561

562

563

564
A) Sarcomere breakdown with overexpression of 3CLPro. hiPSC-CMs transduced with adenovirus overexpressing Strep-Tagged 3CLPro or catalytically inactive C145A control for 48h. Staining shows alpha-actinin (ACTN2), F-actin (phalloidin) and Strep-Tag. DAPI counterstain.

B) Increased sarcomere length with overexpression of 3CLPro. Sarcomeres are stained with ACTN2 to mark Z-disks. Example image of sarcomeres with increased distance.

C) Quantification of sarcomere distance. Sarcomere lengths (Z-disk to Z-disk length, as stained by ACTN2) were quantified for 200 sarcomeres ( $N=2 / 3$ independent experiments? or only 1 exp with 200 sarcomeres?). Statistics shown by Student's t-test $(* * * p=X X X)$.

For all statistics shown, ${ }^{*}=p \leq 0.05,{ }^{* *} p \leq 0.01,{ }^{* * *} p \leq 0.001,{ }^{* * *}=p \leq 0.00001$ 
Fig 4

bioRxiv preprint doi: https://doi.org/10.1101/2022.01.17.476677; this version posted January 19,2022 . The copyright holder for this preprint (which was not certified by peer review) is the author/funder. All rights reserved. No reuse allowed without permission.

A. Obscurin cleavage sites

B.

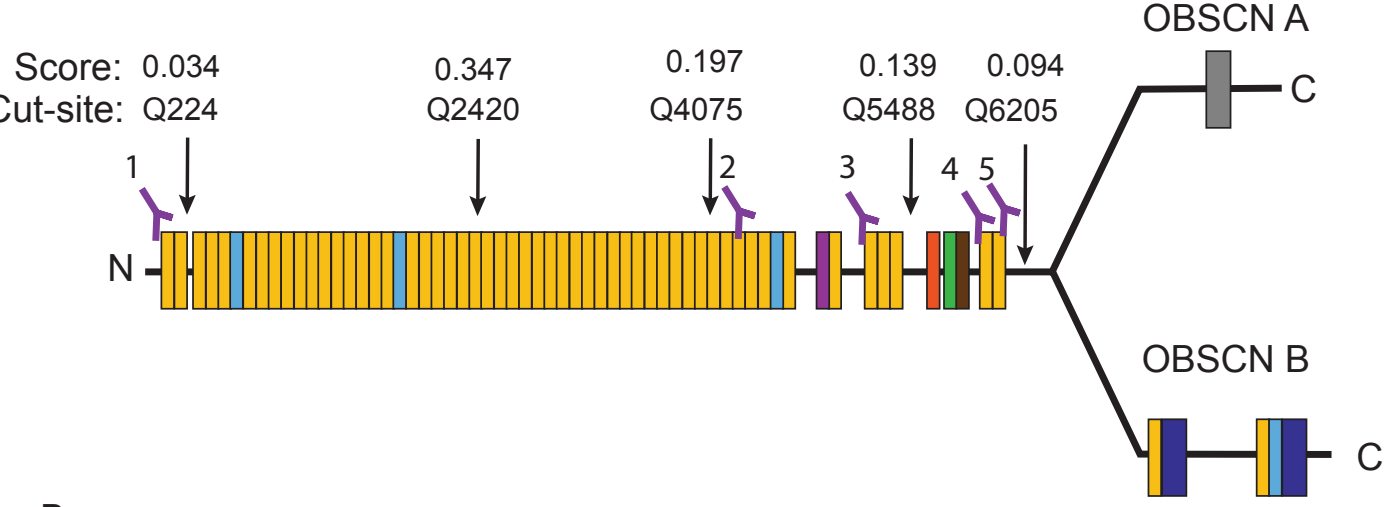

]Ig domain [RhoGEF domain

] FnIII domain $1 \mathrm{PH}$ domain

IQ domain Kinase domain

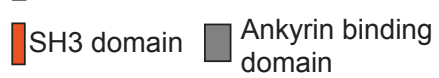
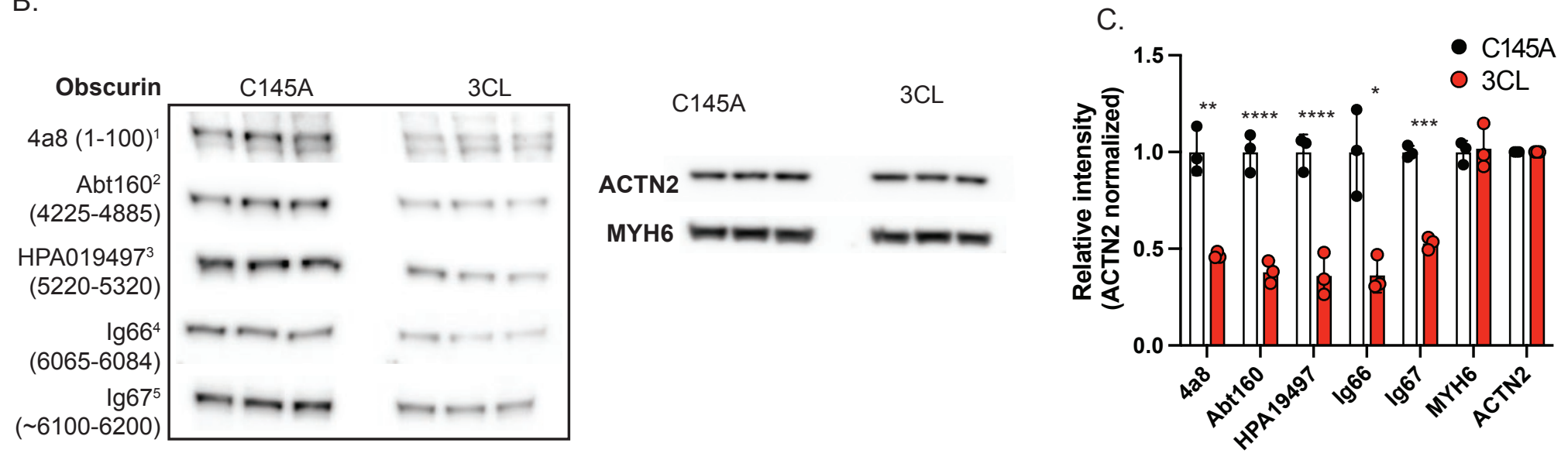

D. Obscurin imaging

Merge
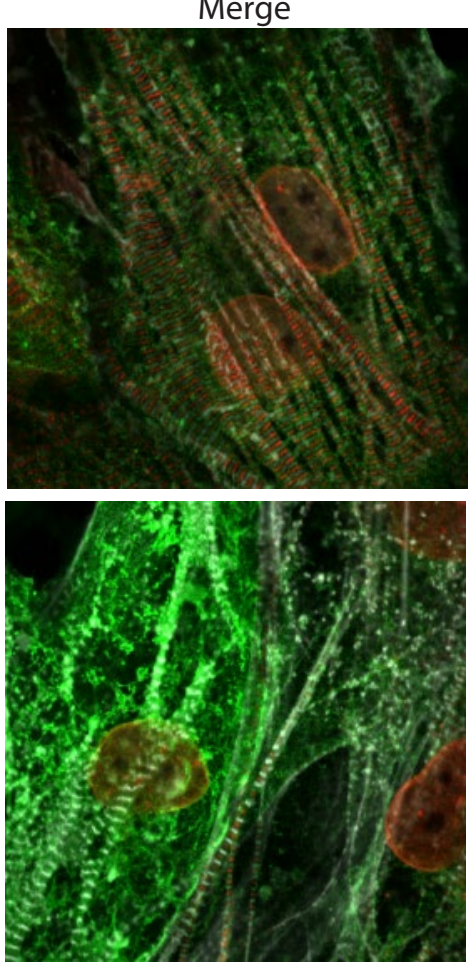

]Strep-Tagll [Obscurin [ACTN2
ACTN2/OBSCN
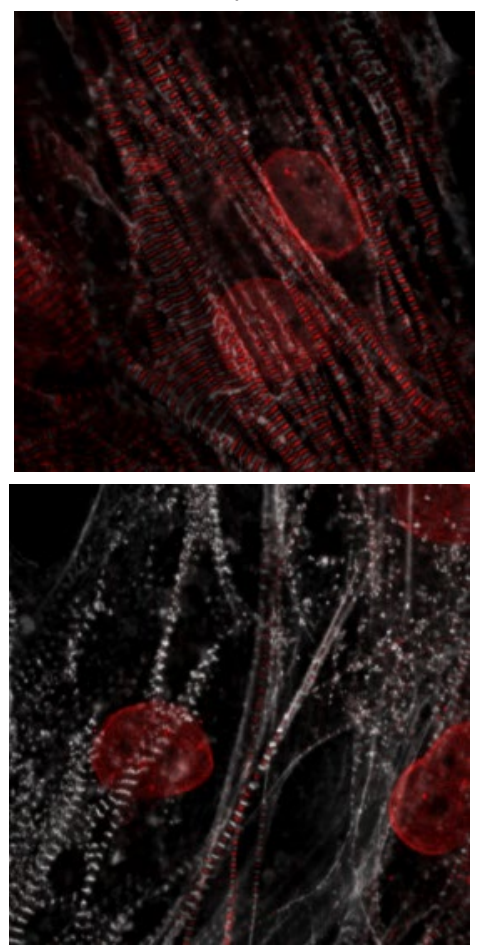

$10 \mu \mathrm{m}$
E.

OBSCN

HPA019497 ${ }^{3}$

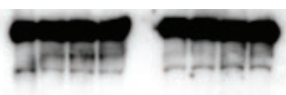

TAB1:C25E9

(Pro381)

${ }^{*}$ Cuts at

Q132, Q144

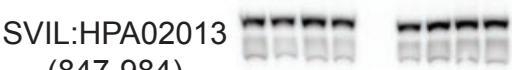

(847-984)

${ }^{*}$ Cut at Q783
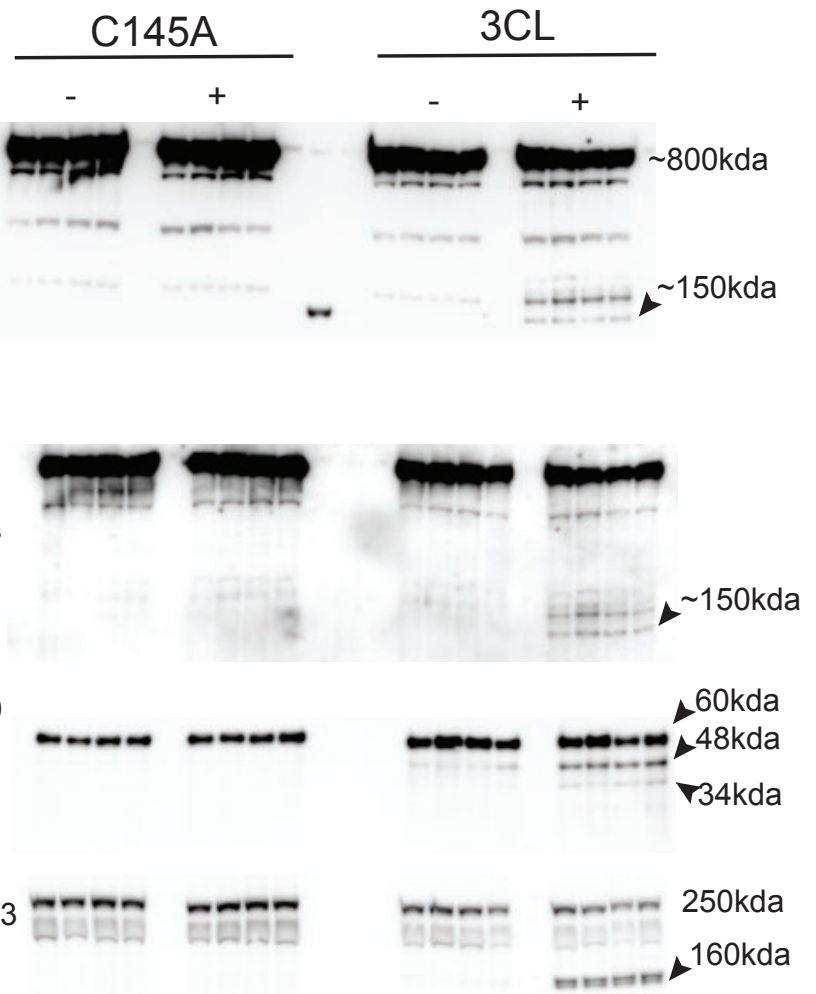
Figure 4

A) Schematic of Obscurin (OBSCN) with overlayed predicted cleavage sites and protein domain. Epitopes regions for antibodies used for this study shown (1-5).

B) Obscurin expression after 72h of 3CLPro or catalytically inactive C145A. Western blots shown for all 5 OBSCN antibodies, as well as ACTN2 and MYH6 controls.

C) Quantification of blots shown in B, normalized by ACTN2 staining on the same membrane. Statistics by Student's t-test.

580 For all statistics shown, ${ }^{*}=p \leq 0.05,{ }^{* *} p \leq 0.01,{ }^{* * *} p \leq 0.001,{ }^{* * * *}=p \leq 0.00001$ 
Fig 5. Obscurin degradation in SAR-COV2 infection

A.

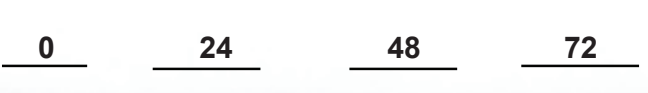

$\mathrm{Abt} 160^{2}$

(4225-4885)

HPA0194973

(5220-5320)
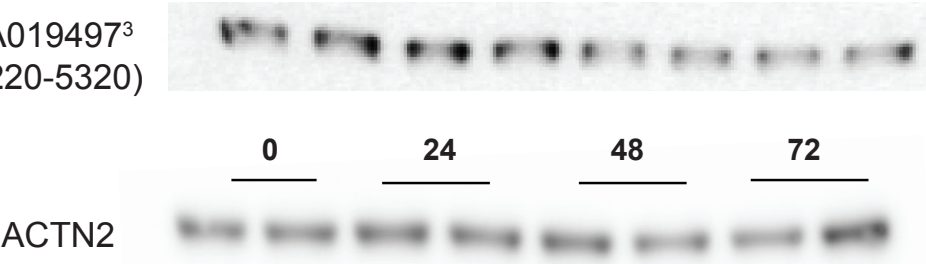

MYH7

MYH6

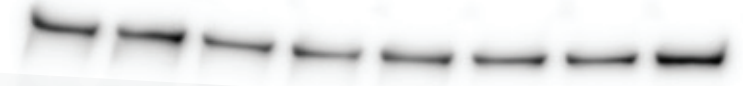

$14-3-3$

Nucleocapsid

C.
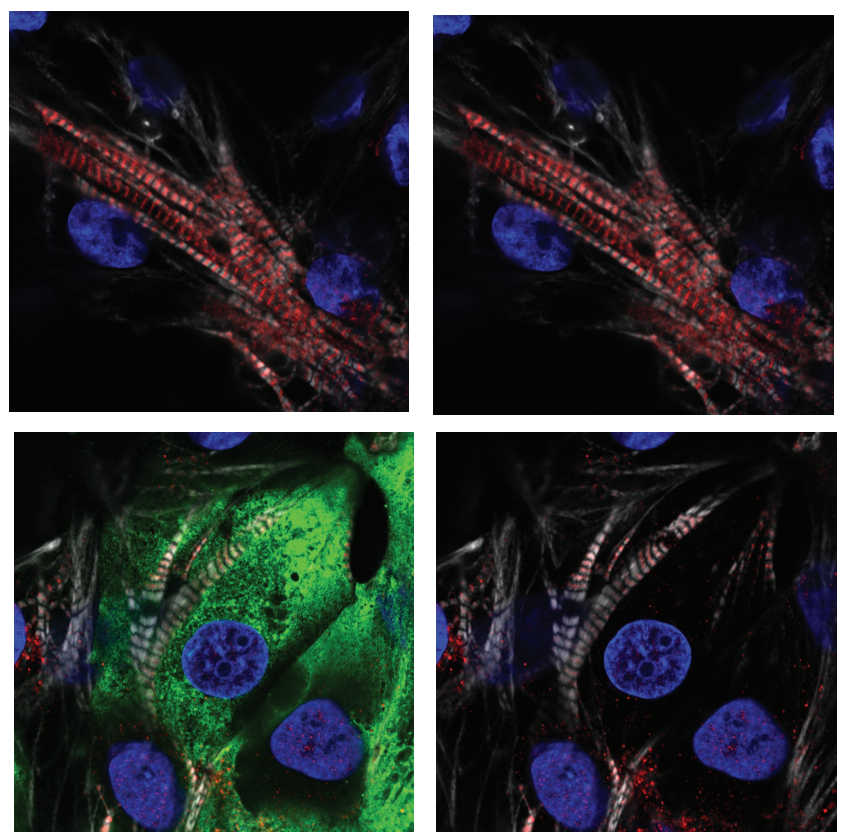

B.

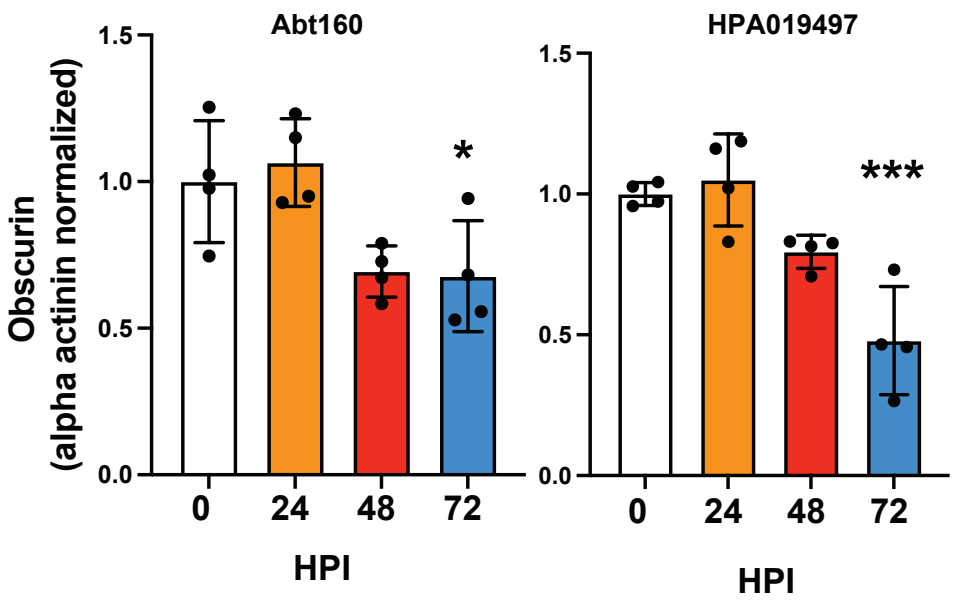

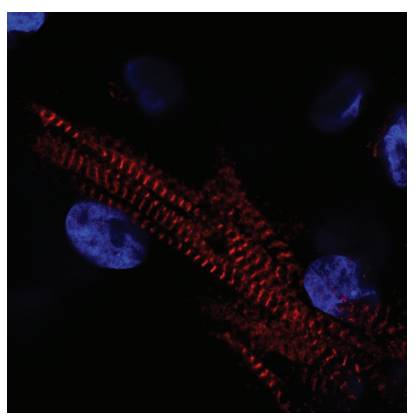
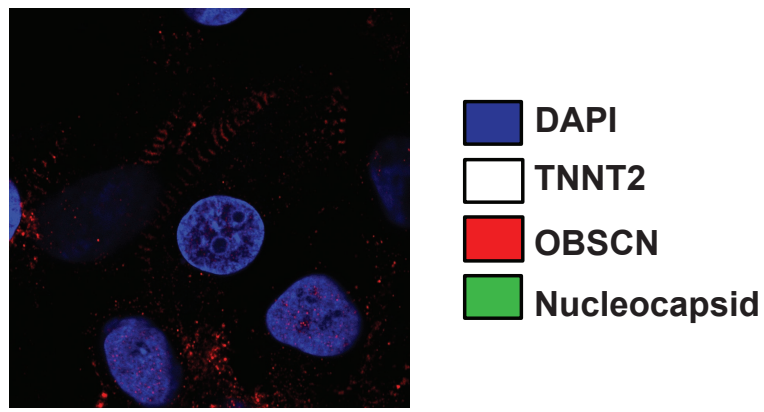

$10 \mu \mathrm{m}$ 
581 Figure 5

A) Western blots of WTC-11c hPSC-CMs infected with SARS-CoV-2 at $5 \mathrm{MOI}$ (Multiplicity of infection) after 24, 48 and $72 \mathrm{~h}$.

B) Quantification of OSCN staining normalized for ACTN2 (per blot). Shown are $n=2$ for each time point for 2 different hPSC-CMs cell lines (WTC-11c and H7) for a total of $n=4$. Statistics calculated by one-way ANOVA with Tukey's post-hoc test.

C) Immunocytochemistry for OBSCN in WTC-11c hPSC-CMs at 48 HPI with 5 MOI SARSCoV-2. TNNT2 used as a counterstain for sarcomeres, and nucleocapsid staining performed to identify infected cells.

For all statistics shown, ${ }^{*}=p \leq 0.05,{ }^{* *} p \leq 0.01,{ }^{* * *} p \leq 0.001,{ }^{* * * *}=p \leq 0.00001$ 


\section{Supplemental Figure 1}

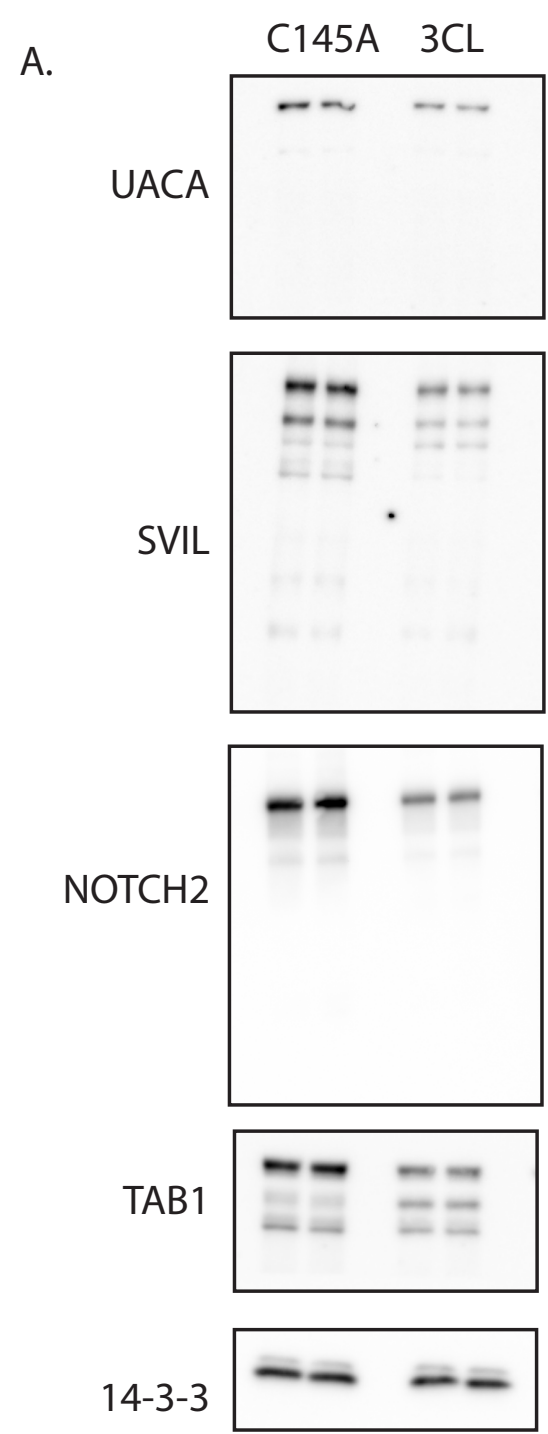

B.

C145A $3 C L$

(C-terminal fragment)

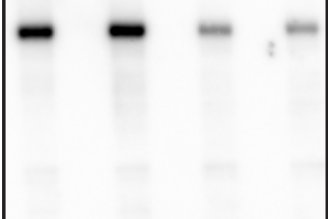

b-Actin

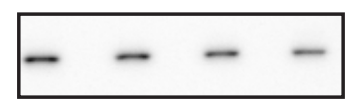

$3 C L$

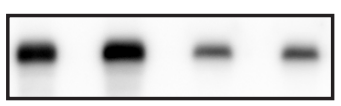


A) Western blots of candidate cleavage targets. 48h overexpression of 3CLPro or C145A in 293 T cells.

596

597

B) Western blot for the C-terminal cleavage fragment of NOTCH1 following $48 \mathrm{~h}$ overexpression in 293T cells. 


\section{Methods}

\section{Bioinformatic prediction of 3CLpro cleavage sites}

In brief, $\mathrm{P} 1$ positions ( $\mathrm{Q}, \mathrm{M}$ or $\mathrm{H}$ ) were first identified across the human proteome. 8 amino acid peptides were generated centered at this position, corresponding to P5 - P3' positions in the $3 C L P r o$ consensus sequence. Scores were generated by multiplying the relative efficiency values published by Chuck, et al for the SARS-COV (2003) 3CLPro. All sites with a score $>0$ (i.e., those that did not have an "ND" in any of the positions from P5- P3') were captured. The full code for this program will be shared upon publication.

\section{In vitro cleavage assays}

In vitro cleavage assays were performed with purified 3CLPro protein and assay buffer from BPS Bioscience. Protease was added at $1 \mathrm{uM}$ concentration, and recombinant protein targets at an approximate ratio of $2 \mathrm{ug}$ target/1ug 3CLPro. Recombinant proteins were purchased commercially: NOTCH1 (Origene, Cat\# TP762041), CDH6 (ACROBiosystem, Cat\# CA6-H5229), CDH20 (R\&D, Cat\# 5604-CA-050). Purified human alpha-thrombin was purchased from Haematologic Technologies (Cat\# 50-883-435).

\section{Cell Culture}

For 3CLPro overexpression assays, human induced pluripotent (hIPSC) ventricular cardiomyocytes were purchased from NCardia. Cells were plated at $\sim 150,000$ cells/24-well on fibronectin coated (Sigma, F1131), glass bottomed nano-patterned plates or coverslips (CuriBio, ANFS-0024). Following 4 days of maturation, cells were transduced with adenovirus (Vector Biolabs) to induce expression of 3CLPro or catalytically inactive C145A, and lysates collected at 48 or $72 \mathrm{~h}$ in TU buffer.

\section{Human induced-pluripotent stem cells culture and differentiation}

Human induced-pluripotent stem cells (WTC11c hiPSCs, gifted by Dr. Bruce Conklin, Gladstone Institutes, San Francisco) were maintained in complete mTeSR Plus (Stem Cell Technologies) and cultured on Matrigel-coated dished at $0.17 \mathrm{mg} / \mathrm{mL}$ (Corning). WTC hiPSCc were passaged as small clumps for maintenance or single cell-like suspension for cardiac differentiation using Versene (Gibco) and mTeSR Plus supplemented with $10 \mu \mathrm{M}$ Y-27632 (Tocris). Cardiac differentiation was perfomed as previsoudly described (PMID: 33657418). Briefly, WTC hiPSCs were seeded at 1,000 cells/cm² using mTeSR1 Plus and $10 \mu \mathrm{M}$ Y-27632 on Matrigel-coated dishes. After $24 \mathrm{~h}$, media was replaced with mTeSR Plus supplemented with $1 \mu \mathrm{M}$ Chiron 99021 (Cayman) to prime the cells for differentiation. Mesoderm induction (Day 0) was performed with $3 \mu \mathrm{M}$ Chiron 99021 in RPMI-1640 media (ThermoFisher) supplemented with $500 \mu \mathrm{g} / \mathrm{mL}$ BSA (Sigma-Aldrich) and $213 \mu \mathrm{g} / \mathrm{mL}$ ascorbic acid (Sigma-Aldrich), named RBA media. After $48 \mathrm{~h}$ (Day 2), cells were treated with RBA media supplemented with $2 \mu \mathrm{M}$ WNT-C59 (Selleckchem). On day 4, media was change with RBA only and cells were incubated for an additional $48 \mathrm{~h}$. From day 6 until day 13, hiPSC-derived cardiomyocytes (hiPSC-CMs) were maintained in RPMI1640 supplemented with B-27 supplement (ThermoFisher). Heat-shock was performed at 42C for $30 \mathrm{~min}$ and on day 14, hiPSC-CMs were dissociated using 0.5\% Trypsin (Gibco) and cryopreserved in Cryostore CS10 (Sigma). 


\section{HiPSC-CMs infection}

All experiments using live virus were performed in the Biosafety Level 3 (BSL-3) facility at the University of Washington in compliance with the BSL-3 laboratory safety protocols (CDC BMBL 5th ed.) and the recent CDC guidelines for handling SARS-CoV-2. Before removing samples from BSL-3 containment, samples were inactivated by Thiourea buffer or $4 \%$ paraformaldehyde, and the absence of viable SARS-CoV-2 was confirmed for each sample by plaque assays described in the next section.

Frozen hiPSC-CMs were thawed in RPMI 1640 supplemented with B27 supplements, $10 \mu \mathrm{M}$ Y27632 and 5\% FBS. After 24 h, media was replaced with RPMI 1640 supplemented with B27 supplements only. A total of 200.000 hiPSC-CMs were seeded three days after thawing in Matrigel-coated 24-wells plate using RPMI 1640 supplemented with B27 supplements, $10 \mu \mathrm{M} \mathrm{Y-}$ 27632 and 5\% FBS. Media was changed after $24 \mathrm{~h}$ and infection was performed as previously described (PMID: 33657418). Briefly, hiPSC-CMs were quickly washed with DPBS and incubated with SARS-CoV-2 at 5 multiplicity of infection (MOI) diluted in DMEM only (Gibco) for $1 \mathrm{~h}$ at 37 C. Mock-control hiPSC-CMs were treated with DMEM only. Media was then replaced with RPMI 1640 supplemented with B27 supplements and samples were collected at $48 \mathrm{~h}$ after infection. For MG132 treated samples, after viral absorption, media was replaced with RPMI 1640 supplemented with B27 supplements and $1 \mu \mathrm{M}$ of MG132 (DMSO was used for control).

\section{SARS-CoV-2 preparation and titer.}

SARS-Related Coronavirus 2, Isolate USA-WA1/2020 (SARS-CoV-2) was obtained from BEI Resources (NR-52281). Virus propagation and titer was performed in VERO cells (USAMRIID) as described in PMID: 33657418. Briefly, VERO cells were maintained in DMEM supplemented with $10 \%$ FBS, $100 \mathrm{U} / \mathrm{mL}$ penicillin, and $100 \mathrm{U} / \mathrm{mL}$ streptomycin and incubated with either 0.1 MOI (virus propagation) or serial dilution of conditioned media (titer) for $1 \mathrm{~h}$ at 37C in DMEM only media for viral absorption. For viral propagation, conditioned media was harvested and aliquots were store in -80C. For titer, 10-fold serial dilutions of conditioned media (either from VERO cells or hiPSC-CMs) were incubated on VERO cells for $1 \mathrm{~h}$ at 37C. A 1:1 mixture of cellulose suspension (Sigma) and DMEM containing 4\% heat-inactivated FBS, L-glutamine, 1X antibiotic-antimycotic (Gibco), and $220 \mathrm{mg} / \mathrm{mL}$ sodium pyruvate was layered on top of the cells and incubated at $37 \mathrm{C}$ for $48 \mathrm{~h}$. Cellulose layer was then removed and cells were stained with $10 \%$ paraformaldehyde and stained with $0.5 \%$ crystal violet solution in $20 \%$ ethanol. Plaques were counted, and the virus titer in the original sample was assessed as plaque-formation unit per $\mathrm{mL}$ (PFU/mL).

\section{Lysate preparation and Western blotting}

For western blotting of 293T cell lysates, traditional RIPA buffer was used to lyse cells. Lysates were normalized for cell concentration by BCA assay, and denatured by addition of Laemmli buffer (BioRad) at 95C for 5 minutes. Samples were then run on tris-glycine gels, followed by transfer onto PVDF membranes. Samples were blocked for 45 minutes with $5 \%$ milk in TBS + $0.1 \%$ Tween-20, and stained overnight at $4 \mathrm{C}$ in Pierce protein-free blocking buffer (ThermoScientific). 
For cardiomyocyte lysates, cells at equivalent densities were first lysed in thiourea denaturing buffer (TU buffer: $8 \mathrm{M}$ urea, $2 \mathrm{M}$ thiourea, 50mM Tris- $\mathrm{HCl} \mathrm{pH} 7.5,3 \% \mathrm{SDS}, 75 \mathrm{mM}$ DTT). Following incubation in TU buffer for 5 minutes, an equivalent volume of $50 \%$ glycerol was added to lysates for a final concentration of $4 \mathrm{M}$ urea, $1 \mathrm{M}$ thiourea, $25 \mathrm{mM}$ Tris $\mathrm{HCl} \mathrm{pH} \mathrm{7.5,} 1.5 \% \mathrm{SDS}, 25 \%$ glycerol and 37.5mM DTT and stored at -80C until western blotting. Samples were not heated so as to avoid urea decomposition, and subsequent carbamylation of proteins from cyanate ions. Phenol red powder was added directly to lysates for visualization These denaturing, highly reducing conditions were crucial for solubilization of sarcomeric proteins. Lysates were run directly on tris-glycine or tris-acetate gels, transferred to PVDF membrane, and stained as described above.

\section{Imaging}

Imaging was performed on a Zeiss LSM 710 confocal microscope at 63X. For sarcomere quantification, lengths of 400 sarcomeres were quantified for $n=3$ biological replicates. Images assignments were blinded during quantification.

\section{Antibodies}

The following antibodies were used for western blotting. Dilutions were 1:1000 unless specified otherwise.

Protein
Alpha actinin (EA-53)
Alpha actinin (EP2529Y)
MYH6
MYH7
Notch1
Notch1
Notch2
Obscurin
Obscurin
Obscurin
Obscurin
Obscurin
Obscurin
Obsl1
SARS-COV/COV2 3CLPro
SARS-COV2 nucleocapsid
SARS-COV2 nucleocapsid
StrepMAB classic
StrepMAB classic-HRP
SVIL
TAB1
Thrombin
Tnnt2 (13-11)

Company
Abcam
Abcam
Sigma Aldrich
Sigma Aldrich
EmdMillipore
Abcam
CST
Gift from Dr. Kontragianni-Konstantapoulos
Gift from Dr. Kontragianni-Konstantapoulos
EmdMillipore
EmdMillipore
EmdMillipore
Sigma (Prestige)
Abcam
Cell Signaling
GeneTex
R \& D Systems
IBA LifeSciences
IBA LifeSciences
Sigma (Prestige)
CST
Novus Biologicals
BD

Cat\#
ab9465
ab68167
HPA001349
M8421
MAB5352, clone mn1a
ab52627, EP1238T
5732, clone D76A6

ABT160
MABT332, Clone 4a8
MABT126, clone 510A
HPA019497
ab204075
51661
GTX135357
MAB10474
2-1507-001
NC9789296
HPA020138
3226, clone C25E9
NBP1-58268
564766

ab9465

68167

PA001349

AB5352, clone mn1

ab52627, EP1238T

5732, clone D76A6 
bioRxiv preprint doi: https://doi.org/10.1101/2022.01.17.476677; this version posted January 19, 2022. The copyright holder for this preprint (which was not certified by peer review) is the author/funder. All rights reserved. No reuse allowed without permission.

Tnnt2-647 conjugate

UACA
BD

BethylLaboratories
565744

A301-383A

\section{Statistics}

Statistics were calculated with Prism9. 